Document downloaded from:

http://hdl.handle.net/10251/99493

This paper must be cited as:

Guijarro, L.; Pla, V.; Vidal Catalá, JR.; Naldi, M. (2017). Game Theoretical Analysis of Service Provision for the Internet of Things Based on Sensor Virtualization. IEEE Journal on Selected Areas in Communications. 35(3):691-706. doi:10.1109/JSAC.2017.2672239

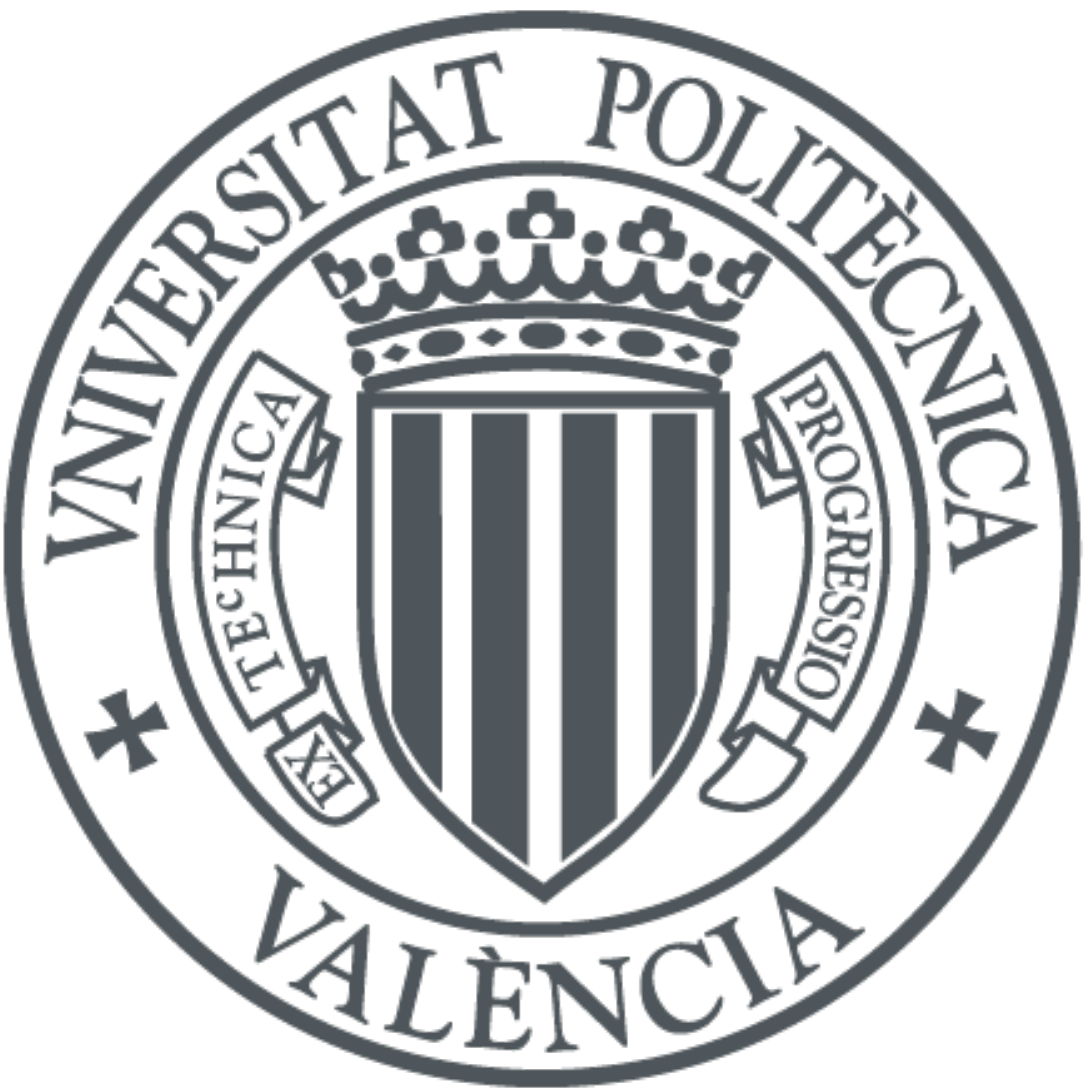

The final publication is available at

http://doi.org/10.1109/JSAC.2017.2672239

Copyright Institute of Electrical and Electronics Engineers

Additional Information 


\title{
Game Theoretical Analysis of Service Provision for the Internet of Things Based on Sensor Virtualization
}

\author{
Luis Guijarro, Vicent Pla, Jose R. Vidal and Maurizio Naldi
}

\begin{abstract}
The advent of the Internet of Things (IoT) is expected to bring major benefits to a wide range of areas. However, the successful deployment of the IoT calls for the existence of sustainable and well-understood business models. In this paper, we propose and analyze a business model for a likely scenario in the IoT, which is made up of WSNs, service providers and users. The service providers compete against each other in the intermediation between the virtualized WSNs and the users that benefit from enhanced services built on the sensed data. The service providers pay to the WSNs for the data and charge the users for the service. The model is analyzed by applying oligopoly theory and game theory, the conditions for the existence and uniqueness of the Nash equilibrium are established, and the equilibrium and the social optimum are obtained. Our results show that the business model is sustainable, provided that the users' sensitivity to the value-to-price ratio is not negligible and, in this situation, the number of active service providers is upper bounded by a value that depends on the sensitivity and the market size. Furthermore, the operation of such a market is shown to efficiently use the information provided by the WSNs; and, when compared to the social optimum, to produce an increase in users' and service providers' surpluses, but a reduction in WSNs' surplus.
\end{abstract}

Index Terms-Game theory, service provision, wireless sensor networks, oligopoly, social welfare

\section{INTRODUCTION}

The "Internet of Things" (IoT) is one of the hottest topics being debated today across industries worldwide. The estimates of the number of smart objects in homes, offices, factories, vehicles and elsewhere are 50 billion by 2020 , up from 12.5 billion in 2010 [1]. Although smart objects are becoming omnipresent, the fact is that the market for services related to these objects is immature. The commercial success of sensorbased services needs that both the appropriate market structure and corresponding pricing schemes be well understood [2].

Wireless sensor network-based service provision is a likely scenario for IoT. In this paper we propose and analyze a wireless sensor network-based service business model in order to contribute to the understanding of the economics of this scenario.

The proposed model consists of autonomous Wireless Sensor Networks (WSNs) that sense data and sell the data to one

This work has been supported by the Spanish Ministry of Economy and Competitiveness through project TIN2013-47272-C2-1-R.

L. Guijarro, V. Pla, and J. R. Vidal are with ITACA, Universitat Politècnica de València, Spain (e-mail: \{lguijar, vpla, jrvidal\}@upv.es)

M. Naldi is with Università di Roma Tor Vergata, Italy (e-mail: maurizio.naldi@uniroma2.it) or more service providers. Each service provider sets up a platform that captures the sensing data, builds the service and provides the service to the users; and it takes care of bundling the solutions, sets the tariffs, bills the customers and provides customer care [1].

Our study models a scenario of what is known as "Sensing as a Service" [3], where providers build services based on sensing data collected by third parties. There is a dominant trend in the economy in general and specifically in the internetbased service provision whereby companies specialize either on service provision or on infrastructure operation; our work is related to this trend. Among all scenarios covered by the term "Sensing as a Service", the scenarios that are amenable to our model are those where it makes sense to pay for the sensing data collected by WSNs. In these scenarios, the WSNs are deployed and owned by entities interested in collecting revenues that cover or help cover the deployment and operating costs of the sensor infrastructure. On the other hand, there are IoT services that do not fit onto the above paradigm, such as smart water services, where the infrastructure is vertically integrated by a utility company that both provides the service and owns the sensor infrastructure. And there are IoT services that would not be amenable to our model, like those based on wearable devices, since individuals, who are the owners of their wearable WSN, are currently not ready to engage in multi-party data sharing agreements. An example would be the following. WSNs may be deployed by high street stores, restaurants and pubs to monitor the movement of customers in and out their premises. This data may be aggregated to build a real-time map of pedestrian density [4], and a service build on geotagged pedestrian density information may be provided to users interested in targeting high density pedestrian spots, e.g., taxi cabs looking for potential riders or out-of-home advertisers running highly targeted campaigns. The data may be supplied with different time and/or spatial resolution and may bear different delays and redundancy, which will translate into different information rates, and ultimately into different qualities of service for the users. The users will be willing to pay a higher price for a better service quality, so that there is room for a competition in both information rates and prices.

The proposed business model assumes a scenario where more than one service provider operate in the market. Service providers are assumed that neither own nor operate the WSNs. The access of the service providers to the WSN-sensed data is implemented through the virtualization of the WSNs [5]. Virtualization is a well-established concept that allows the 
abstraction of actual physical computing resources into logical units, enabling the efficient usage by multiple independent users. Adapted to WSNs, it can allow the efficient utilization of WSN deployment, as multiple applications will be able to coexist on the same virtualized WSN. Virtualizing WSNs brings many benefits, among which we would like to emphasize the elimination of tight coupling between services/applications and WSN deployments, allowing the definition on flexible roles in a business model [6].

The proposed business model is formalized using an analytic model based on microeconomics, specifically on oligopoly theory [7] - as far as the competition between the service providers is concerned, although concepts from discrete-choice analysis are borrowed for the users modeling. Game theory is extensively used for the model and the analysis of the competition between the service providers. The solution procedure is mainly analytical, although numerical non-linear optimization is used in some parts of this paper.

The model incorporates some specifics related to the WSNs operation, such as the influence of the information rate over the user utility, and over the price paid to the WSNs. It also borrows some fundamentals from microeconomics.

There are some studies that discuss which requirements a sustainable business model should comply with in an Internet of Things scenario [8] [9], but few of them have approached this issue formally as our paper.

The authors proposed a business model for the service provision in a WSN scenario in [10]. The focus in the work was specifically to test the feasibility of a pricing mechanism that emulated a two-sided market scenario. But the analysis did not progress beyond a monopoly scenario. Likewise, the authors proposed also an intermediation-based business model in [11] and in [12], analyzing it just in a scenario with one service provider. The present paper, however, succeeds in analyzing the more realistic scenario of competition and it has a broader scope than [10]. Reference [13] provides a survey on the pricing schemes for IoT services and proposes a business model where the provider intermediates between sensors and users, like in our work. Several providers are modeled, but no competition is allowed, which differs from our work. Actually, the goal of [13] is to analyze whether providers will cooperate in offering their IoT service as a bundle or not, and if so, how to optimize the bundled subscription fee.

Interestingly, [14] models the competition in prices in the provision of IoT services, as in our paper. The theoretical framework is information economics, which departs from ours. The model is simple, since the information source is binary, but the approach is novel and promising because it can be applied to model time-sensitive information and information reselling.

There are also proposals of business models in cloud service provision, where service providers compete for users and for virtual resources from infrastructure providers [15]. The setting in this work exhibits some parallelisms with our scenario. However, there are specifics of cloud service provisioning that are different from our scenario, e.g., pricing the on-spot virtual computing resources, which is a crucial point in popular implementations, such as Amazon Web Services.
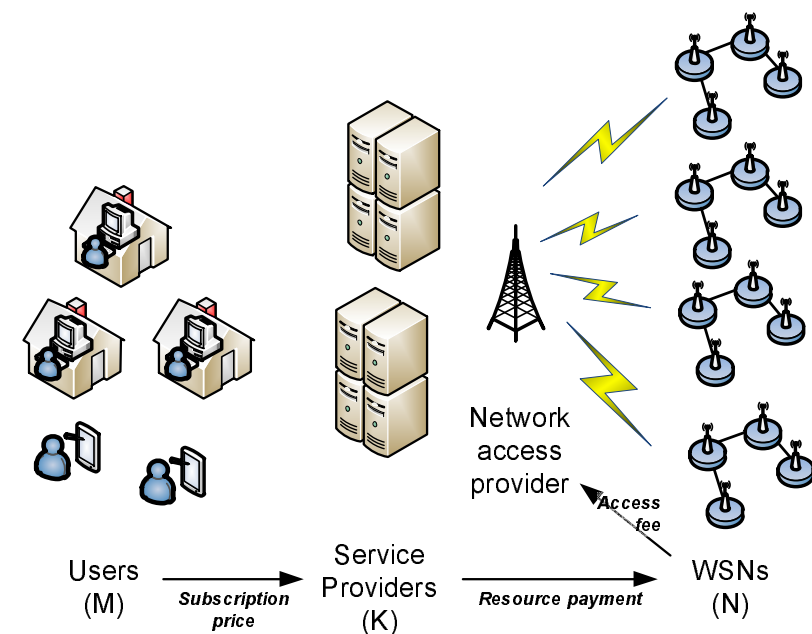

Fig. 1. Scenario model.

Finally, there are studies that focus instead on the hurdles faced by IoT deployments that may compromise the feasibility of the business models supporting them. Reference [16] discusses the CAPEX requirements, and provides examples of IoT deployments that either exhibit CAPEX gains or require small CAPEX. These issues are relevant at large time scales, whereas our work focuses on mechanisms, e.g., pricing, that operate at shorter time scales.

The main contributions of this paper are the following:

- We propose a business model for service provision in the Internet of Things and show that is sustainable-i.e. an equilibrium with active service providers earning positive profits exists-provided that the user sensitivity to the sensing-rate-to-price ratio is not negligible. And in this situation the number of active service providers is upper bounded by a value that depends on the sensitivity and on the market size.

- An exhaustive analysis of the equilibria that the competition yields is conducted and the conditions for their existence and uniqueness are derived.

- The surpluses at the equilibrium for the different actorsWSNs, service providers and users-are obtained and compared with those at the social optimum.

The structure of this paper is as follows. Section II describes the model. Section III solves the game and determines the existence and uniqueness conditions for the Nash equilibrium. Section IV presents the results and, finally, Section V draws some conclusions.

\section{MODEL DESCRIPTION}

The scenario modeled in this paper is shown in Fig. 1 and the details of the payment flows are provided in Figs. 2, 3 and 4. The scenario comprises $N$ wireless sensor networks, $K$ service providers, $M$ users, and one or more network access providers. Next we describe the details of the model for each of these agents. A summary of the notation used in this paper is given in Table I. 
TABLE I

SUMMARY OF NOTATION

Eq. page

\begin{tabular}{|c|c|c|c|}
\hline \multicolumn{4}{|l|}{ WSNs } \\
\hline Number of WSNs & \multicolumn{2}{|l|}{$N$} & 2 \\
\hline Number of connected WSNs (random variable) & \multicolumn{2}{|l|}{$\mathcal{N}$} & 3 \\
\hline Information rate generated by WSN $j$ & \multicolumn{2}{|l|}{$r_{j}$} & 3 \\
\hline Random variable from which the $r_{j}$ 's are drawn & \multicolumn{2}{|l|}{$\mathcal{R}$} & 3 \\
\hline $\begin{array}{l}\text { Price per unit of information rate charged by } \\
\text { WSNs }\end{array}$ & \multicolumn{2}{|l|}{$a$} & 3 \\
\hline $\begin{array}{l}\text { Network access fee payed by each WSN to a } \\
\text { Network Access Provider }\end{array}$ & \multicolumn{2}{|l|}{$f$} & 3 \\
\hline Profit of WSN $j$ & $\Pi_{j}$ & (1) & 3 \\
\hline Expected number of connected WSNs & $n$ & (3) & 3 \\
\hline \multirow[t]{2}{*}{$\begin{array}{l}\text { Expected value of the information rate generated } \\
\text { by connected WSNs }\end{array}$} & $r$ & (4) & 3 \\
\hline & \multicolumn{2}{|l|}{$\begin{array}{l}r_{\max } \\
R_{m}, f_{m}\end{array}$} & $\begin{array}{l}3 \\
4\end{array}$ \\
\hline $\begin{array}{l}\text { Expected aggregated information rate provided } \\
\text { by all connected WSNs }\end{array}$ & & $(5)$ & 3 \\
\hline Inverse supply function & $a\left(R_{T}\right)$ & (10) & 4 \\
\hline WSNs surplus & $\Pi_{\mathrm{WSNs}}$ & (17) & 6 \\
\hline \multicolumn{4}{|l|}{ Service providers (SPs) } \\
\hline Number of SPs & \multicolumn{3}{|l|}{$K$} \\
\hline $\begin{array}{l}\text { Aggregated information rate that SP } i \text { obtains } \\
\text { from the WSNs }\end{array}$ & \multicolumn{3}{|l|}{$R_{i}$} \\
\hline Priced charged by SP $i$ to each of its subscribers & \multirow{2}{*}{\multicolumn{3}{|c|}{$p_{i}$}} \\
\hline Profit of SP $i$ & & & 5 \\
\hline Aggregate profit of all SPs in the symmetric case & $\Pi_{\mathrm{SPS}}^{(s)}$ & (17) & 6 \\
\hline \multicolumn{4}{|c|}{ Users } \\
\hline Number of users & $M$ & & 2 \\
\hline $\begin{array}{l}\text { Objective part of the utility that a user obtains } \\
\text { from SP } i\end{array}$ & $v_{i}$ & $(12)$ & 4 \\
\hline Sensitivity parameter & \multicolumn{2}{|l|}{$\mu$} & 4 \\
\hline $\begin{array}{l}\text { Subjective part of the utility that user } u \text { obtains } \\
\text { from SP } i\end{array}$ & \multicolumn{2}{|l|}{$\kappa_{u, i}$} & 4 \\
\hline Users' sensitivity parameter & \multicolumn{2}{|l|}{$\alpha$} & 5 \\
\hline $\begin{array}{l}\text { Number of users that subscribe to the } i^{t h} \text { service } \\
\text { provider }\end{array}$ & \multicolumn{3}{|l|}{$m_{i}$} \\
\hline $\begin{array}{l}\text { Fraction of users that subscribe to the } i^{t h} \text { service } \\
\text { provider }\end{array}$ & $\sigma_{i}$ & $(13)$ & 5 \\
\hline Users' surplus & $\Pi_{\text {users }}$ & (14) & 5 \\
\hline Users' surplus in the symmetric case & $\Pi_{\text {users }}^{(s)}$ & $(15)$ & 5 \\
\hline \multicolumn{4}{|l|}{ Analysis, results and auxiliary notation } \\
\hline $\begin{array}{l}\text { Vector of zeros (ones) of the appropriate dimen- } \\
\text { sion }\end{array}$ & \multicolumn{2}{|l|}{$0 /(1)$} & 7 \\
\hline $\begin{array}{l}\text { CDF of the continuous uniform distribution in } \\
{[0,1]}\end{array}$ & \multicolumn{2}{|l|}{$\Psi(\cdot)$} & 4 \\
\hline $\begin{array}{l}\text { Upper bound of } k \text { that appears in certain condi- } \\
\text { tions }\end{array}$ & $\eta_{M}$ & $(31)$ & 7 \\
\hline Set of the integer numbers that lie in $\left[\eta_{m}, \eta_{M}\right)$ & $\mathcal{K}$ & & 8 \\
\hline Social Welfare & \multicolumn{3}{|l|}{ SW } \\
\hline \multirow[t]{2}{*}{ Social Welfare in the symmetric case } & $\mathrm{SW}^{(s)}$ & (39) & 14 \\
\hline & $\begin{array}{l}\boldsymbol{p}_{-i}, \boldsymbol{R}_{-i} \\
\mathcal{D}_{i}\left(\boldsymbol{R}_{-i}\right) \\
\left(\boldsymbol{p}^{*}, \boldsymbol{R}^{*}\right)\end{array}$ & (19) & $\begin{array}{l}6 \\
6 \\
6\end{array}$ \\
\hline Indices of the SPs entering the market & $\mathcal{I}(\boldsymbol{p}, \boldsymbol{R})$ & (26) & 7 \\
\hline Number of SPs entering the market & $k \equiv k(\boldsymbol{p}, \boldsymbol{R})$ & (27) & 7 \\
\hline & $A(k)$ & (28) & 7 \\
\hline & $\beta$ & (30) & \\
\hline $\begin{array}{l}\text { Unique solution in }(0,1) \text { to } A(k)(1-x)^{3}- \\
\left(1-\frac{x}{\beta}\right)^{2}=0\end{array}$ & $z_{0}(k)$ & & 7 \\
\hline & $I_{\text {cond }}(x)$ & (36) & 8 \\
\hline Value at which $I_{\text {cond }}(x)$ crosses zero & $\eta_{m}$ & & 8 \\
\hline
\end{tabular}

\section{A. Wireless Sensor Networks}

There exist $N$ WSNs. Each WSN senses information, which is bundled by the service providers in order to compose useful services to the users.

WSN $j$ generates information at a rate $r_{j}$. We define information rate as the net rate of sensing information that the WSNs supply to the service providers. This information flow, which we propose to measure in net bits per second (nbps), is assumed to comply with the QoS requirements included in the service level agreement between the WSNs and the service providers, e.g., latency, reliability. This approach is in line with the research that assesses the quality of information and the value of information in sensor networks [17]. According to this approach, we have considered that the existence of possible redundancies in the data sensed by neighboring WSNs is already taken into account in the value of the information rate they supply to service providers.

Each WSN is paid an amount $a \cdot r_{j}$, where $a$ is the price per information rate unit and is the same for all WSNs, and it pays a price $f$ to a Network Access Provider (NAP), which may be different from the service provider. All prices will be measured in generic currency units (cu). While the link of the sensors to the WSN gateway will obviously be wireless, the connection of the WSN gateway to the NAP will typically be through a fixed broadband line, for which the flat rate is the typical choice - see, e.g., the survey by the Open Technology Institute in [18] and the supporting rationale in [19]. It is to be noted that, for the purpose of analysis, any additional fixed costs that the WSN may incur may be incorporated in the fee $f$. We assume that the number of WSNs is sufficiently high so that each one is assumed to take prices $a$ and $f$ as given.

Therefore, provided that WSN $j$ enters the market, it will get the profit

$$
\Pi_{j}=a \cdot r_{j}-f,
$$

as depicted in Fig. 2 . Note that the profit $\Pi_{j}$ cannot be negative since WSN $j$ will not enter the market if $a \cdot r_{j}-f<0$, as it is discussed next.

The WSNs are heterogeneous in their information rates $r_{j}$. We model this heterogeneity by assuming that the values $r_{j}(j=1, \ldots, N)$ are samples of a random variable $\mathcal{R}$ distributed in the interval $\left[0, r_{\max }\right]$. In the general case this random variable will be described by a probability distribution function $F_{\mathcal{R}}(x)=\mathrm{P}(\mathcal{R} \leq x)$. WSN $j$ will enter the market if $\Pi_{j} \geq 0$, which is an event with probability

$$
\mathrm{P}(a \mathcal{R}-f \geq 0)=\mathrm{P}\left(\mathcal{R} \geq \frac{f}{a}\right)=1-F_{\mathcal{R}}\left(\frac{f}{a}\right) .
$$

The number of connected WSNs, $\mathcal{N}$, is then a random variable and the expected number of connected WSNs, $n$, is given by

$$
n=\mathbb{E}[\mathcal{N}]=N\left(1-F_{\mathcal{R}}\left(\frac{f}{a}\right)\right) .
$$

Additionally, the rate of a connected WSN has an expected value, $r$, which is given by

$$
r=\mathbb{E}\left[\mathcal{R} \mid \mathcal{R} \geq \frac{f}{a}\right]=\frac{f}{a}+\int_{f / a}^{\infty} \frac{1-F_{\mathcal{R}}(x)}{1-F_{\mathcal{R}}(f / a)} \mathrm{d} x .
$$

Given (3) and (4), the expected aggregate rate that all connected WSNs supply, $R_{T}$, is given by

$R_{T}=n \cdot r=N\left(\frac{f}{a}\left(1-F_{\mathcal{R}}\left(\frac{f}{a}\right)\right)+\int_{f / a}^{\infty}\left(1-F_{\mathcal{R}}(x)\right) \mathrm{d} x\right)$. 
Since there are no available statistics on the actual distribution of information rates that can be met across a wide variety of WSNs, in the following we will consider a uniform distribution as the best one to be used when we have no further information, which is supported by their typical use as a noninformative prior in a Bayesian framework [20]. Additionally, the uniform distribution is appropriate for modeling a large degree of heterogeneity among the WSNs. An example of analysis for the monopoly case under the Zipf distribution, which adopts a specific hierarchical set of rates for the WSNs, is conducted in [21].

If $\mathcal{R}$ is uniformly distributed in the interval $\left[0, r_{\max }\right]$, the expressions (2)-(5) take the form

$$
\begin{aligned}
& \mathrm{P}(a \mathcal{R}-f \geq 0)=1-\Psi\left(\frac{f_{m}}{a}\right), \\
& n=\mathbb{E}[\mathcal{N}]=N\left(1-\Psi\left(\frac{f_{m}}{a}\right)\right), \\
& r=\mathbb{E}\left[\mathcal{R} \mid \mathcal{R} \geq \frac{f}{a}\right]=\frac{r_{\max }}{2}\left(1+\Psi\left(\frac{f_{m}}{a}\right)\right), \\
& R_{T}=n \cdot r=R_{m}\left(1-\Psi^{2}\left(\frac{f_{m}}{a}\right)\right),
\end{aligned}
$$

where $f_{m}=f / r_{\max }, R_{m}=N r_{\max } / 2$ and $\Psi(\cdot)$ is the cumulative distribution function of the uniform distribution in $[0,1]$.

The expression for $R_{T}$ given in (9) can be interpreted as an aggregate supply function, since it gives the total amount of rate supplied by the WSNs for a given unit price $a$.

Regarded as a function of $a$, (9) can be inverted and the resulting function, $a:\left[0, R_{m}\right) \rightarrow\left[f_{m},+\infty\right)$, is

$$
a\left(R_{T}\right)=\frac{f_{m}}{\sqrt{1-\frac{R_{T}}{R_{m}}}} .
$$

The above expression can be interpreted as the unit price that should be posted to the WSNs in order to obtain an aggregate information rate $R_{T}$, i.e., it is the inverse supply function. This allows to model the set of connected WSNs in an aggregate manner, as depicted in Fig. 3. This representation will be used hereafter in this paper.

Finally, the WSNs' surplus is defined as the sum of the profits of all (connected) WSNs, and can be computed as follows

$$
\begin{aligned}
\Pi_{\mathrm{WSNs}}=\mathbb{E}\left[\sum_{j=1}^{N} \Pi_{j}\right]= & N \mathbb{E}\left[\Pi \mid \mathcal{R} \geq \frac{f}{a}\right] \mathrm{P}\left(\mathcal{R} \geq \frac{f}{a}\right)= \\
& R_{m} a\left(R_{T}\right)\left(1-\frac{f_{m}}{a\left(R_{T}\right)}\right)^{2}
\end{aligned}
$$

\section{B. Users}

Users are interested in accessing a range of services that the service providers compose using the information they buy from the WSNs.

We use a discrete-choice model for the modeling of the users' choices, which is frequently used in econometrics [22]. Specifically, given a discrete set of options, the utility of a user

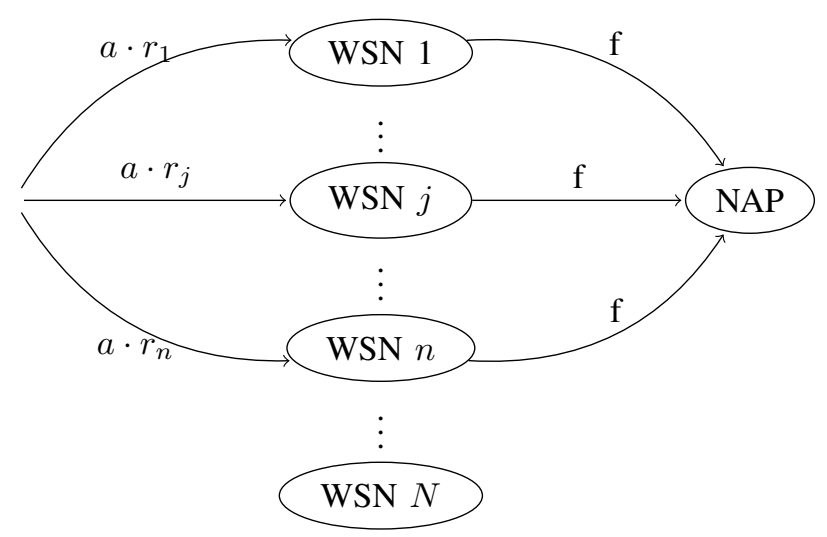

Fig. 2. Individual WSN model and payment flows.

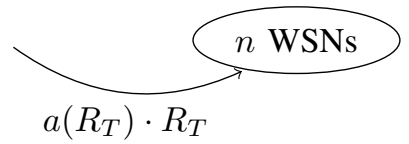

Fig. 3. Aggregate WSN model and payment flows.

$u$ making the choice $i$ is assumed to be equal to $v_{i}+\kappa_{u, i}$ : the term $v_{i}$ encompasses the objective aspects of option $i$ and is the same for all users, while $\kappa_{u, i}$ is an unobserved user-specific value that is modeled on the global level as a random variable. From the distribution of these i.i.d. variables, one can compute the probability that a user selects option $i$, and when the user population size $M$ is sufficiently large, this corresponds to the proportion of users making that choice.

In our model, the user choice is the choice of one of the $K$ service providers, which are indexed by $i=1, \ldots, K$, and we use $i=0$ for the "no-provider" option.

To model the objective part of the users' utility, we assume that each user pays a flat-rate price $p_{i}$ to service provider $i$, and receives an aggregate information rate $R_{i}$. Following [23], we propose

$$
v_{i}=\mu \log \left(\frac{R_{i}}{p_{i}}\right) .
$$

Firstly, the higher the information rate a provider gets, the higher the utility the user derives from the service. This makes sense since services that process more precise data can provide better quality. The dependence is logarithmic, as there is an increasing evidence that user experience and satisfaction in telecommunication scenarios follow logarithmic laws [24]. Secondly, the dependence on the price is through a negative logarithm, instead; or in other words, the ratio $R_{i} / p_{i}$ is proposed to be the relevant magnitude for the utility. Thirdly, $\mu>0$ is a sensitivity parameter. And fourthly, note that the "no-provider" option may be characterized by a ratio $R_{0} / p_{0}$, i.e., this option can be made more or less desirable by tuning this ratio.

To model the unobserved user-specific part of the utility, following the literature on discrete-choice models, we borrow a logit model; specifically, we assume that each user-specific random variable $\kappa_{u, i}$ follows a Gumbel distribution of mean 
0 and parameter $\nu^{1}$. The choice of the Gumbel distribution allows us to obtain a logistic function in the end.

Note that we assume that the users are price-takers, which is a sensible assumption for a sufficiently high $M$.

We define $\sigma_{i}$ as the number of users $m_{i}$ that subscribe to the $i^{t h}$ service provider over the total number of users $M$ - against the other $K-1$ providers and the "no-provider" option. With the users' utility modeled as stated above, it can be shown [25] that

$$
\sigma_{i}=\frac{m_{i}}{M}=\frac{\left(R_{i} / p_{i}\right)^{\alpha}}{\sum_{l=0}^{K}\left(R_{l} / p_{l}\right)^{\alpha}}, \quad i=1, \ldots, K,
$$

where $\alpha=\mu / \nu$ is the users' sensitivity parameter, that is, it models the sensitivity to the information-rate-to-price ratio, and as such, the higher $\alpha$ is, the more sensible the users are to the information rate and the price chosen by each service provider. In the limit, when $\alpha$ tends to infinity, the service provider choosing the highest ratio will get all users; whereas if $\alpha$ is equal to zero all service providers, including the "noprovider", will get exactly the same amount of users.

Finally, the users' surplus is defined as the sum of the utilities of all users, which can be computed as [25]

$$
\Pi_{\text {users }}=\sum_{u=1}^{M} \mathbb{E}\left[\underset{i}{\operatorname{maximize}} v_{i}+\kappa_{u, i}\right] .
$$

For the symmetric case in which the values of $R_{i}$ and $p_{i}$ are the same for all service providers $\left(R_{i}=R_{j}\right.$ and $p_{i}=p_{j}$, $i, j=1, \ldots, K)$, this results in

$$
\Pi_{\mathrm{users}}^{(s)}=M \log \left(\left(\frac{R_{0}}{p_{0}}\right)^{\alpha}+K\left(\frac{R_{i}}{p_{i}}\right)^{\alpha}\right) .
$$

\section{Service providers}

Each Service Provider (SP) performs two basic roles in the model:

- it composes services that are based on the information generated by the WSN;

- it acts as an intermediary between users and WSNs, which allows to decouple the pricing schemes on each side.

There are $K$ SPs competing in providing services to the users and in being procured by the WSNs. The payment flow is depicted in Fig. 4 and the strategic games played are explained in detail below.

1) Competition in the resource procurement: There are a number of SPs competing to access the information rate resource from the WSNs.

The WSNs are assumed to be price takers and therefore are modeled through the inverse supply function (10).

However, the SPs are not so numerous as to be assumed price takers; on the contrary, they can influence the resource price in two ways: both directly, through its own choice of $R_{i}$, and indirectly, through its strategic influence on the choice of each competitor $R_{l}, l \neq i$.

\footnotetext{
${ }^{1}$ The probability density function of the Gumbel distribution of mean 0 and parameter $\nu$ is $f(x)=\frac{1}{\nu} e^{-(x / \nu+\gamma)+e^{-(x / \nu+\gamma)}}$, where $\gamma \approx 0.57721$ is the Euler-Mascheroni constant.
}

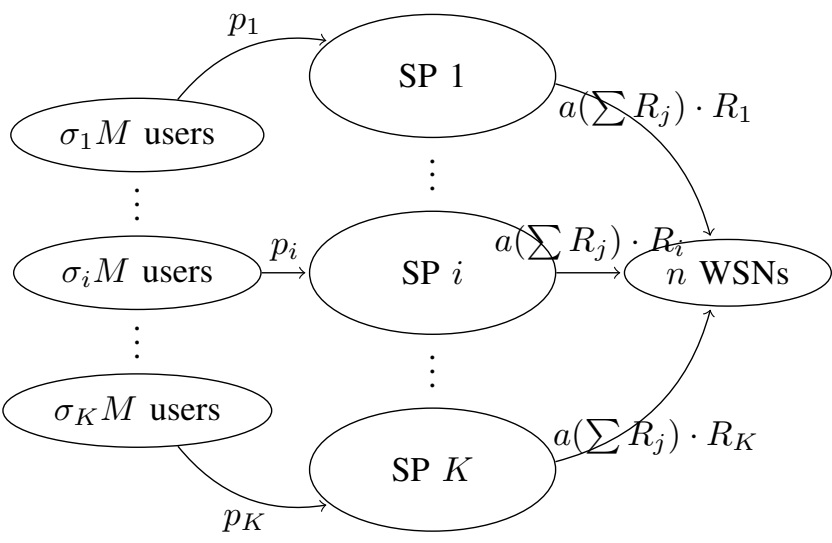

Fig. 4. Full model and payment flows.

The appropriate model for the interaction between the $K$ SPs is therefore an oligopsony. Specifically, we propose a noncooperative simultaneous game with the following characteristics. Each SP is a player. The strategy of the i-th SP is the amount $R_{i}$ of information rate to be procured and the payoff is given by $-a\left(\sum_{j=1}^{K} R_{j}\right) \cdot R_{i}$. Here we note that the price depends on the sum of the choices of each provider, which relies on the following two assumptions. First, that the total information rate procured by the WSNs matches the sum of the SPs' requests. This should be interpreted in the light of the definition of information rate in Section II-A. And second, that the information rate resource procured from the WSNs is not reused among the service providers. The latter assumption is realistic when modeling scenarios where wireless sensors are virtualized, either at a node level or at a network level, in such a way that the memory/CPU/sensing resource of a physical sensor is partitioned and each part is allocated through a virtual sensor node or network to a different application or service provider [6].

2) Competition in the service provision: The same number $K$ of SPs compete against each other in the provision of the service to the $M$ users.

The users are assumed to be price takers and the demand is modeled as described in Section II-B.

The competition between the $K$ SPs, following the same reasoning as above, is modeled as an oligopoly of a price competition type. Specifically, we propose a non-cooperative simultaneous game with the following characteristics. First, each SP is a player. Second, each player's strategy is the following pair of choices: price $p_{i}$ charged to each subscriber, and $R_{i}$. And third, the payoff is given by $p_{i} \cdot m_{i}$, where $m_{i}$ encapsulates the dependence on the strategy of the $K-1$ competitors.

3) Simultaneous competition: The two competition games described above are played by the same $K$ service providers. Actually, the relevant payoff for each provider is the profit, which equals the sum of the payoffs of the two competition games, and results in the following expression:

$$
\Pi_{i}=p_{i} M \sigma_{i}-a\left(\sum_{j=1}^{K} R_{j}\right) R_{i}, \quad i=1, \ldots, K
$$


where $\sigma_{i}$ and $a(\cdot)$ are given by (13) and (10), respectively.

We propose that the two competition games are played simultaneously, instead of sequentially. The rationale is that the choice of $R_{i}$ cannot be regarded as an investment choice or a capital expenditure, but an operational expenditure, and as such is executed within the same time scale as the pricing decision on the users side.

Thus, the combined procurement and service competition game is specified as follows:

- each one of the $K$ service providers is a player;

- each player's strategy is the following pair of choices: price $p_{i}$ and $R_{i}$;

- the payoff is given by (16).

Note that there is a coupling between the two competition games through the payoffs and through the strategies used.

Finally, the total SPs profits, for the special case of identical $R_{i}$ values and identical $p_{i}$ values, is given by:

$$
\Pi_{S P s}^{(s)}=K\left(p_{i} M \sigma_{i}-a\left(K R_{i}\right) R_{i}\right) .
$$

\section{ANALYSIS}

In this section, the game defined in the previous section is solved, that is, the Nash equilibrium is computed and the conditions for existence and uniqueness are provided. As the main result of our analysis, we prove that, when the users' sensitivity is high enough $(\alpha>1)$ and the number of SPs $(K)$ is below a certain upper limit $\left(K<\eta_{M}\right)$, there exists a symmetric equilibrium in which all SPs enter the market (see Propositions 4 and 5). We also show that, for $\alpha>1$ and under some very specific conditions, other equilibria in which not all SPs enter the market may exist. Finally, we also show that when the user's sensitivity is not high enough $(\alpha \leq 1)$ the only possible equilibrium, if any, is one in which no SP enters the market.

Below we make some introductory remarks, and after that we carry out the detailed analysis through a series of propositions and corollaries. In this section, we omit proofs of all propositions and corollaries for the sake of brevity; the detailed proofs can be found in an accompanying document [26]. At the end of this section, in Subsection III-B, we summarize the results of the analysis.

By recalling (13) and (16) we write the general expression of the $i$-th provider's profit

$$
\begin{array}{r}
\Pi_{i}\left(p_{i}, R_{i} ; \boldsymbol{p}_{-i}, \boldsymbol{R}_{-i}\right)=M p_{i} \frac{\left(R_{i} / p_{i}\right)^{\alpha}}{\sum_{j=0}^{K}\left(R_{j} / p_{j}\right)^{\alpha}}-a\left(\sum_{j=1}^{K} R_{j}\right) R_{i} \\
i=1, \ldots, K, \quad \text { (18) }
\end{array}
$$

where $\boldsymbol{p}_{-i}=\left(p_{1}, \ldots, p_{i-1}, p_{i+1}, \ldots, p_{K}\right)$ and $\boldsymbol{R}_{-i}=$ $\left(R_{1}, \ldots, R_{i-1}, R_{i+1}, \ldots, R_{K}\right)$. By abuse of notation we will sometimes denote $\Pi_{i}\left(p_{i}, R_{i} ; \boldsymbol{p}_{-i}, \boldsymbol{R}_{-i}\right)$ simply as $\Pi_{i}\left(p_{i}, R_{i}\right)$ or even $\Pi_{i}$ if such simplified notation is unambiguous.

Note that the model is overparameterized since the $\Pi_{i}$ 's depend on $p_{0}$ and $R_{0}$ through their ratio $R_{0} / p_{0}$ and not through their individual values. Thus, we can set $R_{0}=1$. Moreover, without any loss of generality, we can also set $p_{0}=1$, which is equivalent to a normalization of all monetary/utility values by $p_{0}$. In other words, this is as if we were using a currency unit so that the price $p_{0}$ expressed in that currency is 1 .

We need to consider the case in which one or more SPs decide not to enter the market. The situation in which SP $j$ does not enter the market corresponds to $\left(p_{j}, R_{j}\right)=(0,0)$, which calls for an extension of the definition of $\Pi_{j}$, since (18) is not defined when $p_{j}=0$ for some $j=1, \ldots, K$.

In the redefinition of the SP profits the following aspects are considered:

- If SP $j$ does not enter the market, i.e. $\left(p_{j}, R_{j}\right)=(0,0)$, we should have that $\Pi_{j}=0$, and from the perspective of a different SP $i$ it should be as if SP $j$ did not exist.

- For any $p_{i} \geq 0, \Pi_{i}\left(p_{i}, 0 ; \boldsymbol{p}_{-i}, \boldsymbol{R}_{-i}\right)=$ $\Pi_{i}\left(0,0 ; \boldsymbol{p}_{-i}, \boldsymbol{R}_{-i}\right)=0$, which has a clear interpretation, because if $R_{i}=0$, no user will subscribe to $\mathrm{SP} i$, and then the price $p_{i}$ posted by $\mathrm{SP} i$ is completely irrelevant. Therefore, for all intents and purposes, we can consider all the points $\left(p_{i}, R_{i}\right)$ of the form $\left(p_{i}, 0\right)$ as equivalent to, and thus represented by, the point $(0,0)$.

- For an SP it does not make sense to utilize a strategy of the form $\left(0, R_{i}\right)$ with $R_{i}>0$, since $\Pi_{i}\left(0, R_{i} ; \boldsymbol{p}_{-i}, \boldsymbol{R}_{-i}\right)=-a\left(\sum_{j=1}^{K} R_{j}\right) R_{i}<$ $\Pi_{i}\left(0,0 ; \boldsymbol{p}_{-i}, \boldsymbol{R}_{-i}\right)=0$. In other words, a point in which $p_{i}=0$ and $R_{i}>0$ could never be an equilibrium since SP $i$ would be better off by playing $(0,0)$.

Now, let $\left(\boldsymbol{p}_{-i}, \boldsymbol{R}_{-i}\right)$ be a fixed pair of $(K-1)$-tuples such that

$$
\begin{aligned}
\left(p_{j}, R_{j}\right) \in\{(0,0)\} \cup\{(0, \infty) \times(0, \infty)\} & \\
& j=1, \ldots, i-1, i+1, \ldots, K
\end{aligned}
$$

and $\sum_{j \neq i} R_{j}<R_{m}$. The function $\Pi_{i}\left(p_{i}, R_{i} ; \boldsymbol{p}_{-i}, \boldsymbol{R}_{-i}\right), \quad i=$ $1, \ldots, K$, can be defined in the domain

$$
\mathcal{D}_{i}\left(\boldsymbol{R}_{-i}\right)=\{(0,0)\} \cup\left\{(0, \infty) \times\left(0, R_{m}-\sum_{j \neq i} R_{j}\right)\right\}
$$

as

$$
\begin{aligned}
& \prod_{i}\left(p_{i}, R_{i} ; \boldsymbol{p}_{-i}, \boldsymbol{R}_{-i}\right)= \\
& \begin{cases}0 & \text { if } p_{i}=0 \\
M p_{i} \frac{\left(R_{i} / p_{i}\right)^{\alpha}}{K}-a\left(\sum_{j=1}^{K} R_{j}\right) R_{i} & \text { otherwise. }\end{cases} \\
& 1+\sum_{\substack{j=1 \\
p_{j} \neq 0}}^{K}\left(R_{j} / p_{j}\right)^{\alpha}
\end{aligned}
$$

The discussion above is summarized in the following proposition that gives necessary, but not sufficient, conditions for a point to be an equilibrium.

Proposition 1: If $\left(\boldsymbol{p}^{*}, \boldsymbol{R}^{*}\right)=\left(\left(p_{1}^{*}, \ldots, p_{K}^{*}\right),\left(R_{1}^{*}, \ldots, R_{K}^{*}\right)\right)$ is a (Nash) equilibrium point then, for each $i=1, \ldots, K$, one of the following two conditions must be met

1) $\left(p_{i}^{*}, R_{i}^{*}\right)=(0,0)$, i.e., the SP does not enter the market;

2) $\left(p_{i}^{*}, R_{i}^{*}\right)$ is a maximum of $\Pi_{i}\left(p_{i}, R_{i} ; \boldsymbol{p}_{-i}, \boldsymbol{R}_{-i}\right)$ in $(0, \infty) \times\left(0, R_{m}^{*}-\sum_{j \neq i} R_{j}^{*}\right)$, which implies

$$
\frac{\partial \Pi_{i}}{\partial p_{i}}\left(p_{i}^{*}, R_{i}^{*} ; \boldsymbol{p}_{-i}, \boldsymbol{R}_{-i}\right)=\frac{\partial \Pi_{i}}{\partial R_{i}}\left(p_{i}^{*}, R_{i}^{*} ; \boldsymbol{p}_{-i}, \boldsymbol{R}_{-i}\right)=0 \text {. }
$$


The following proposition provides necessary and sufficient conditions for $(\mathbf{0}, \mathbf{0})$ being an equilibrium ${ }^{2}$. Note that the point $(\mathbf{0}, \mathbf{0})$ represents the situation in which no SP enters the market.

Proposition 2:

1) If $0 \leq \alpha<1$, then $(\mathbf{0 , 0})$ is not an equilibrium.

2) If $\alpha=1$, then $(\mathbf{0}, \mathbf{0})$ is an equilibrium iff $f_{m} / M \geq 1$.

3) If $\alpha>1$, then $(\mathbf{0}, \mathbf{0})$ is an equilibrium iff $f_{m} / M \geq$ $(\alpha-1)^{1-1 / \alpha} \cdot \alpha^{-1}$.

a) If $f_{m} / M \geq 1$, then $(\mathbf{0 , 0})$ is an equilibrium for any $\alpha \in(1, \infty)$.

b) If $f_{m} / M<1 / 2$, then $(\mathbf{0}, \mathbf{0})$ is not an equilibrium for any $\alpha \in(1, \infty)$.

c) If $1 / 2 \leq f_{m} / M<1$, there exist $\alpha_{1}$ and $\alpha_{2}$ ( $1<$ $\left.\alpha_{1} \leq 2 \leq \alpha_{2}\right)$ such that $(\mathbf{0}, \mathbf{0})$ is an equilibrium if $\alpha \in\left[\alpha_{1}, \alpha_{2}\right]$ and it is not otherwise.

Now we seek equilibrium points other than $(\mathbf{0}, \mathbf{0})$. For this we first look for pairs $\left(p_{i}, R_{i}\right) \neq(0,0)$ that meet the second condition in Proposition 1.

To obtain the partial derivatives of (16) we first write the derivatives of $\sigma_{i}$ in (13) as

$$
\begin{aligned}
\frac{\partial \sigma_{i}}{\partial p_{i}} & =-\frac{\alpha}{p_{i}} \sigma_{i}\left(1-\sigma_{i}\right), \\
\frac{\partial \sigma_{i}}{\partial R_{i}} & =\frac{\alpha}{R_{i}} \sigma_{i}\left(1-\sigma_{i}\right),
\end{aligned}
$$

and from these

$$
\begin{aligned}
& \frac{\partial \Pi_{i}}{\partial p_{i}}=M \sigma_{i}\left(1-\alpha\left(1-\sigma_{i}\right)\right) \\
& \frac{\partial \Pi_{i}}{\partial R_{i}}=M p_{i} \frac{\alpha}{R_{i}} \sigma_{i}\left(1-\sigma_{i}\right)-a\left(\sum_{j=1}^{K} R_{j}\right)-a^{\prime}\left(\sum_{j=1}^{K} R_{j}\right) R_{i}
\end{aligned}
$$

where the function $a(\cdot)$ was defined in (10).

Below we deal separately with the case where $0 \leq \alpha \leq 1$ and the more complex and richer one where $\alpha>1$.

Proposition 3: If $0 \leq \alpha \leq 1$ there are no equilibria different from $(\mathbf{0}, \mathbf{0})$.

The following corollary, which follows immediately from Proposition 2 and Proposition 3, summarizes the situation regarding possible equilibria when $0 \leq \alpha \leq 1$.

Corollary 1:

1) If $0 \leq \alpha<1$, there are no equilibrium points.

2) If $\alpha=1$, then $(\mathbf{0}, \mathbf{0})$ is the only possible equilibrium equilibrium point, and it exists iff $f_{m} / M \geq 1$.

Unlike the case $\alpha \leq 1$, when $\alpha>1$ equilibrium points other than $(\mathbf{0}, \mathbf{0})$ can exist. Proposition 4 provides some structural properties of these equilibrium points, as well as necessary conditions for their existence. Before formulating this proposition, we need to introduce some definitions and notations.

\footnotetext{
${ }^{2}$ The notation/symbol $\mathbf{0}$ represents a vector of zeros of the appropriate dimension. Likewise, 1 represents a vector of ones of the appropriate dimension.
}

For a given point $(\boldsymbol{p}, \boldsymbol{R}) \neq(\mathbf{0}, \mathbf{0})$, we denote by $k(\boldsymbol{p}, \boldsymbol{R})$, or simply $k$, the number of SPs entering the market, and by $\mathcal{I}(\boldsymbol{p}, \boldsymbol{R})$ the set of indices of these SPs. Formally,

$$
\begin{aligned}
\mathcal{I}(\boldsymbol{p}, \boldsymbol{R}) & =\left\{j \in\{1, \ldots, K\} \mid p_{j} \neq 0, R_{j} \neq 0\right\}, \\
k(\boldsymbol{p}, \boldsymbol{R}) & =|\mathcal{I}(\boldsymbol{p}, \boldsymbol{R})| .
\end{aligned}
$$

Let us also introduce

$$
\sqrt{A(k)}=\frac{M}{f_{m}} \frac{\alpha-1}{\alpha}\left(\frac{\alpha}{\alpha-1}-k\right)^{1 / \alpha} .
$$

Furthermore, if $A(k)>1$ let $z_{0}(k)$ represent the unique solution in $(0,1)$ to

$$
A(k)(1-x)^{3}-\left(1-\frac{x}{\beta}\right)^{2}=0,
$$

where

$$
\beta=\frac{k}{k-1 / 2}>1 \text {. }
$$

Note that, on account of Lemma 1 in [26], $z_{0}(k)$ is well defined.

Proposition 4: Let $\alpha>1$ and let us assume that $\left(\boldsymbol{p}^{*}, \boldsymbol{R}^{*}\right) \neq$ $(\mathbf{0}, \mathbf{0})$ is an equilibrium point. Then

1) The number of SPs that enter the market in this equilibrium point, $k \equiv k\left(\boldsymbol{p}^{*}, \boldsymbol{R}^{*}\right)$, is upper-bounded as

$$
k<\eta_{M},
$$

where $\eta_{M}$ is given by

$$
\eta_{M} \equiv \frac{\alpha}{\alpha-1}-\left(\frac{\alpha}{\alpha-1} \frac{f_{m}}{M}\right)^{\alpha}<\frac{\alpha}{\alpha-1} .
$$

2) The equilibrium strategy, $\left(p_{i}^{*}, R_{i}^{*}\right)$, of all the SPs that enter the market is the same

$$
\left(p_{i}^{*}, R_{i}^{*}\right)=\left(p^{*}(k), R^{*}(k)\right) \quad \text { for all } i \in \mathcal{I}\left(\boldsymbol{p}^{*}, \boldsymbol{R}^{*}\right),
$$

where

$$
\begin{aligned}
R^{*}(k) & =\frac{z_{0}(k)}{k} R_{m}, \\
p^{*}(k) & =\left(\frac{\alpha}{\alpha-1}-k\right)^{1 / \alpha} R^{*}(k) .
\end{aligned}
$$

3) The fraction of users that subscribe to each SP entering the market solely depends on the users' sensitivity, and it does it through the rather simple expression

$$
\sigma_{i}=1-\frac{1}{\alpha} \quad \text { for all } i \in \mathcal{I}\left(\boldsymbol{p}^{*}, \boldsymbol{R}^{*}\right)
$$

Proposition 4 establishes some necessary conditions and characteristics that the equilibrium points different from $(\mathbf{0}, \mathbf{0})$ must have. Note, however, that this proposition provides no information about the existence or the uniqueness of the equilibrium. In other words, Proposition 4 does not rule out the possibility that no such equilibrium exits, or that there exist more than one (each with a different number of SPs entering the market).

Combining Proposition 2, Corollary 1 and Proposition 4 gives the following corollary, which asserts that if the situation when no SP enters the market is an equilibrium then there are no other equilibria. 
Corollary 2: If $(\mathbf{0}, \mathbf{0})$ is an equilibrium then it is the unique equilibrium.

It is worth recalling that so far, in the case $\alpha>1$, we are dealing with just potential equilibrium points. In the next two propositions the required conditions for these potential equilibrium points to be actual equilibria are established. Specifically, the next proposition states that if the total number of SPs $(K)$ is below the upper bound $\eta_{M}$ and all the $K$ SPs enter the market, then the $2 K$-tuple of the form given in Proposition 4 (with $k=K$ ) is indeed an equilibrium point.

Proposition 5: Let $\alpha>1$, and $\eta_{M}, p^{*}(K), R^{*}(K)$ as defined, respectively, in Eqs. (31), (33), (32). If $K<\eta_{M}$, then $\left(\boldsymbol{p}^{*}, \boldsymbol{R}^{*}\right)=\left(p^{*}(K) \cdot \mathbf{1}, R^{*}(K) \cdot \mathbf{1}\right)$ is an equilibrium point.

The next proposition provides, along with Proposition 4, the necessary and sufficient conditions to have a non-trivial equilibrium in which not all the $K$ SPs enter the market.

Proposition 6: Let $\alpha>1$, and $\left(\boldsymbol{p}^{*}, \boldsymbol{R}^{*}\right) \neq(\mathbf{0}, \mathbf{0})$ be a point fulfilling the necessary conditions to be an equilibrium given in Proposition 4. And let $k<K$. Then, $\left(\boldsymbol{p}^{*}, \boldsymbol{R}^{*}\right)$ is an equilibrium point iff

$$
\alpha^{1 / \alpha} \geq \sqrt{A(k)\left(1-z_{0}(k)\right)} .
$$

From a practical perspective, the previous proposition provides limited insight into the values of $k<\eta_{M}$ that meet the condition in (35) and hence yield a non-trivial equilibrium. We now turn our attention to this question so as to obtain a more insightful and operational characterization of the values of $k$ that satisfy (35).

\section{A. Study of the condition given in Proposition 6}

Let us introduce

$$
I_{\text {cond }}(x)=\alpha^{1 / \alpha}-\sqrt{A(x)\left(1-z_{0}(x)\right)},
$$

which is defined in $\left[1, \eta_{M}\right.$ ) and indicates (with a non-negative value) whether $x$ satisfies the condition in (35). Note that, for convenience in our analysis, here we allow $x$ to take noninteger values.

Firstly, we observe that if $x$ is sufficiently close to $\eta_{M}$, then the condition is satisfied. Indeed, it is a simple matter to check that letting $x \rightarrow \eta_{M}^{-}$yields $A(x) \rightarrow 1$ and $z_{0}(x) \rightarrow 0$. Hence, since $\alpha>1$,

$$
\lim _{x \rightarrow \eta_{M}^{-}} I_{\text {cond }}(x)=\alpha^{1 / \alpha}-1>0 .
$$

Secondly, by numerical evaluation we have checked that in the interval $\left[1, \eta_{M}\right)$ the function $I_{\text {cond }}(x)$ is increasing and crosses zero at a value near $\eta_{M}$ (see Fig. 5). Let us denote by $\eta_{m}$ the value at which $I_{\text {cond }}(x)$ crosses zero, i.e., $I_{\text {cond }}\left(\eta_{m}\right)=$ 0 .

Therefore, a point $(\boldsymbol{p}, \boldsymbol{R})$ in which some, but not all, SPs enter the market $(0<k(\boldsymbol{p}, \boldsymbol{R})<K)$ is an equilibrium point iff has the form given in Proposition 4 and

$$
k(\boldsymbol{p}, \boldsymbol{R}) \in\left[\eta_{m}, \eta_{M}\right) .
$$

Figure 6 shows that $\eta_{m} / \eta_{M}$ is always very close to 1 , and that it tends to be even closer as $\alpha$ or $M$ increase.
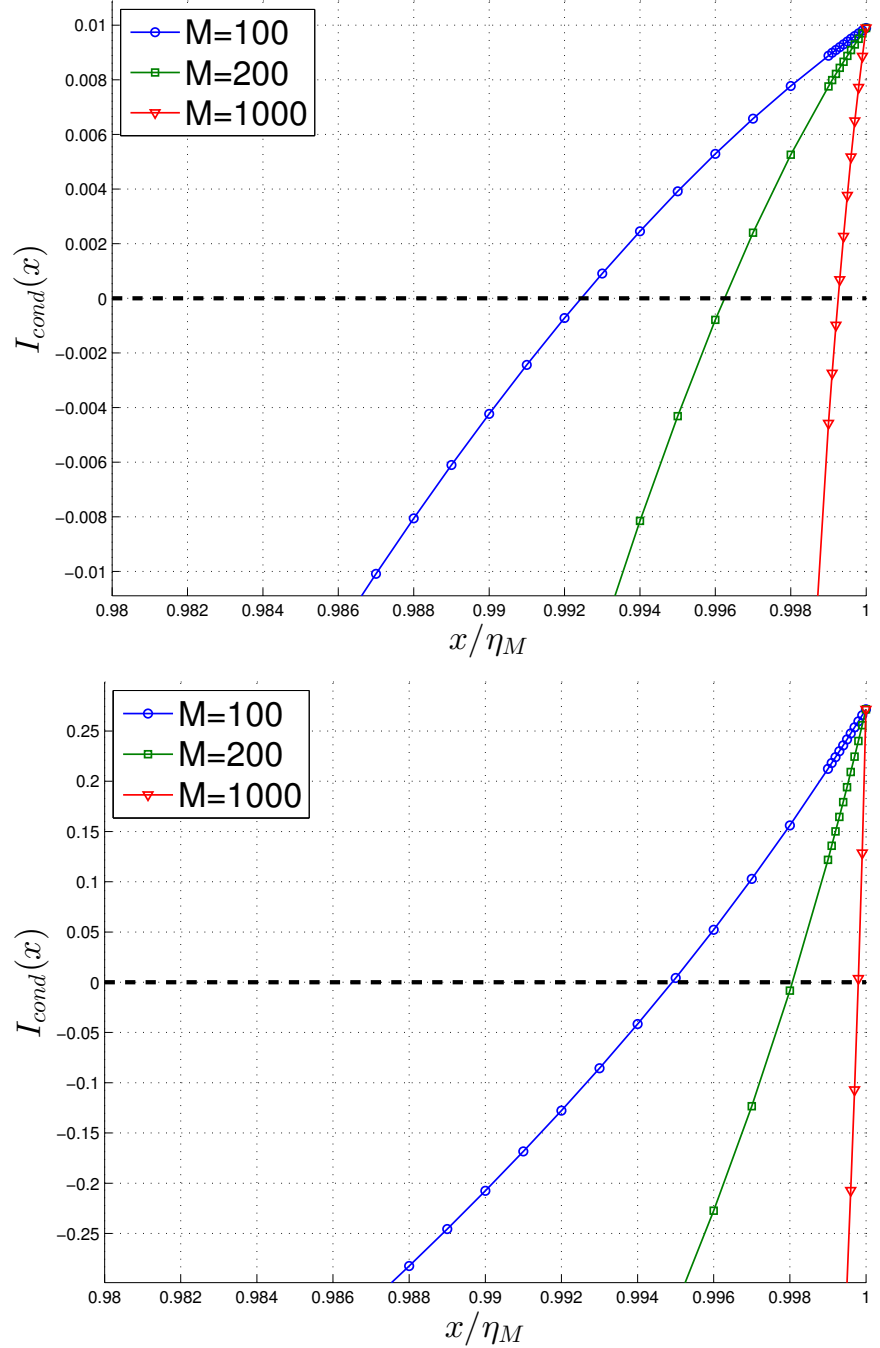

Fig. 5. Graphical representation of $I_{\text {cond }}(x)$ : left, $\alpha=1.01$; right, $\alpha=1.4$

In Fig. 7 we plotted the length of the interval $\left[\eta_{m}, \eta_{M}\right)$ as a function of $\alpha$ (starting at $\alpha=1.005$ ) for different values of $M$. The length of the interval can be arbitrarily large by choosing $\alpha$ close enough to 1 , but falls very rapidly as $\alpha$ grows above 1 (at $\alpha=1.01$ all curves have dropped below 1).

Rather than the length of the interval $\left[\eta_{m}, \eta_{M}\right)$ our interest here is the number of integers it contains; they are closely related, but are not exactly the same thing. Let $\mathcal{K}=\left[\eta_{m}, \eta_{M}\right) \cap$ $\mathbb{Z}$ be the set of the integer numbers that lie in $\left[\eta_{m}, \eta_{M}\right)$, and $|\mathcal{K}|$ its cardinality $\left(0 \leq|\mathcal{K}|=\left\lfloor\eta_{M}\right\rfloor-\left\lceil\eta_{m}\right\rceil+1\right)$. According to our previous observations from Fig. 7, if $\alpha$ is close enough to 1 , the set $\mathcal{K}$ will contain many integers. On the other hand, when $\alpha$ grows and we have $\eta_{M}-\eta_{m} \leq 1$, then $\left(\eta_{m}, \eta_{M}\right)$ will contain none or one integer number, i.e., $|\mathcal{K}| \leq 1$. Indeed, for values of $\alpha$ beyond a certain threshold (which is not far above from 1 ) we have $\mathcal{K}=\varnothing$, except for a series of short intervals that get sparser as $\alpha$ increases.

\section{B. Summary of the results of the analysis}

Our analysis has shown that $\alpha \geq 1$ is a necessary condition for an equilibrium to exist. We can interpret this condition as 


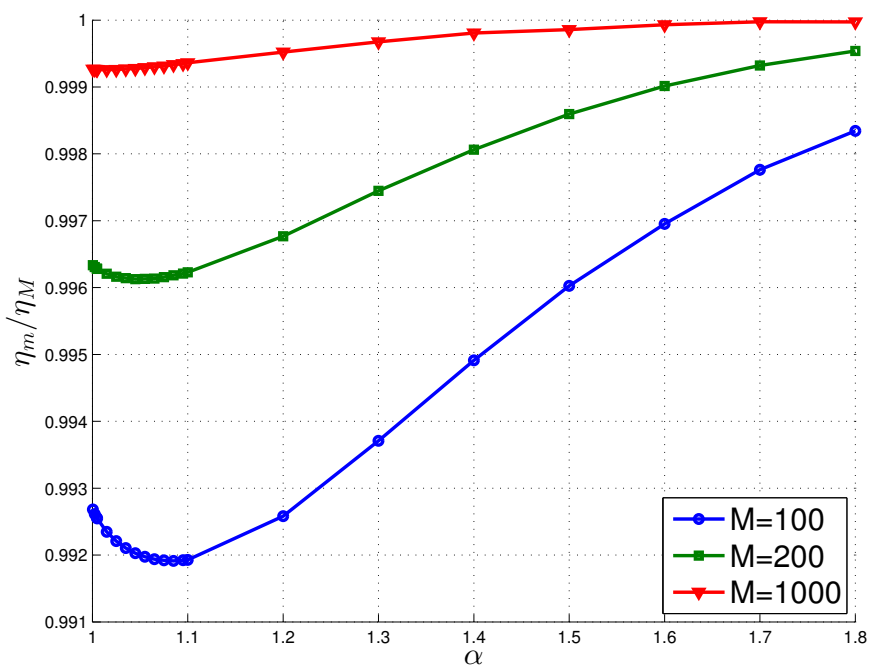

Fig. 6. Position of $\eta_{m}$ relative to $\eta_{M}$.

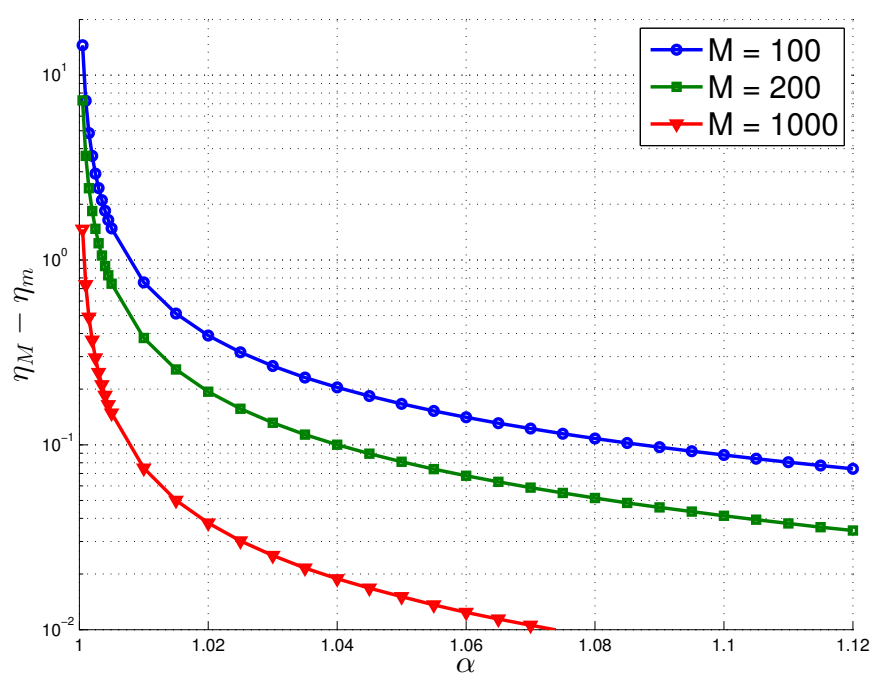

Fig. 7. Length of the interval $\left[\eta_{m}, \eta_{M}\right)$.

follows: the users need be sensitive enough to the ratio $R_{i} / p_{i}$ so that each provider's strategy may attract users in an effective manner. Otherwise, the interaction between the providers will not have any equilibrium.

Table II summarizes the possible equilibrium situations when $\alpha>1$, derived in Propositions 5 and 6. The table reports the number of SPs that, for each situation, will enter the market in equilibrium. We recall that in all cases all SPs entering the market will play the same strategy. When $\eta_{M} \leq 1$ no SP enters the market in the equilibrium (i.e., $(\mathbf{0}, \mathbf{0})$ is the only equilibrium point). However, this situation is not expected to occur in real settings since it requires a relatively high value for $f_{m} / M$ (at least 0.5 ; see Proposition 2), which amounts to a very low number of end users, $M$.

When $\eta_{M}>1$ there are two possible situations depending on whether the number of SPs, $K$, is below or above the thresholds value $\eta_{m}$. If $K \leq\left\lceil\eta_{m}\right\rceil$ there exists a unique equilibrium in which all the $K$ SPs enter the market.
TABLE II

NUMBER OF SPS, OUT OF A TOTAL OF $K$, THAT IN EQUILIBRIUM ENTER THE MARKET WHEN $\alpha>1$

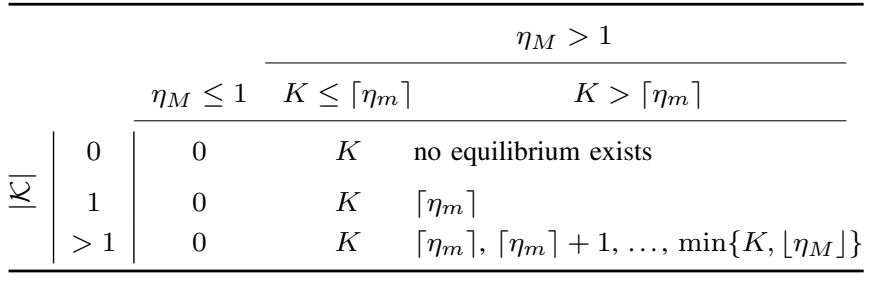

When $K>\left\lceil\eta_{m}\right\rceil$ (and $\eta_{M}>1$ ) we need to consider three different cases depending on the number of integer numbers there are in the interval $\left[\eta_{m}, \eta_{M}\right)$, i.e. $|\mathcal{K}|$ :

1) If $|\mathcal{K}|=0$ no equilibrium exists.

2) if $|\mathcal{K}|=1$ there is one equilibrium type in which $\left\lceil\eta_{m}\right\rceil$ out of $K$ SPs enter the market. Note that here the equilibrium is unique up to the selection of the $\left\lceil\eta_{m}\right\rceil$ indices.

3) If $|\mathcal{K}|>1$ there are several possible equilibrium types with a different number of SPs entering the market in each of them $\left(\left\lceil\eta_{m}\right\rceil,\left\lceil\eta_{m}\right\rceil+1, \ldots\right)$. Again, for each of these types the equilibrium is unique up to the selection of indices.

Our previous numerical analysis showed that the third case $(|\mathcal{K}|>1)$ can only arise for a very narrow range of values of $\alpha$ near 1; $(1<\alpha<1.01$ or narrower for the studied scenarios; see Fig 7). Otherwise, only the first and second case (i.e, no equilibrium or one equilibrium type with some SPs outside the market) are possible.

Finally, all the results in this section about the existence of equilibria can be summarized as follows:

- $0 \leq \alpha<1$ : There are no equilibrium points (Corollary 1 ).

- $\alpha=1:(\mathbf{0}, \mathbf{0})$ is an equilibrium iff $f_{m} / M \geq 1$ (Corollary 1).

- $\alpha \geq 1$ : Two types of equilibrium, which are mutually exclusive (Corollary 2), are possible.

1) $(\mathbf{0}, \mathbf{0})$ is an equilibrium iff $f_{m} / M \geq(\alpha-$ $1)^{1-1 / \alpha} / \alpha$ (Proposition 2).

2) If $\left(\boldsymbol{p}^{*}, \boldsymbol{R}^{*}\right) \neq(\mathbf{0}, \mathbf{0})$ is an equilibrium point in which $k \equiv k\left(\boldsymbol{p}^{*}, \boldsymbol{R}^{*}\right)$ SPs enter the market, $0<$ $k \leq K$, then it is necessary, but no sufficient, that $k<\eta_{M}$ (Proposition 4).

a) If $k=K$ the condition given above is sufficient as well, i.e., $\left(\boldsymbol{p}^{*}, \boldsymbol{R}^{*}\right)$ is an equilibrium iff $k=$ $K<\eta_{M}$ (Proposition 5).

b) If $k<K$ an additional condition is required for sufficiency, i.e., $\left(\boldsymbol{p}^{*}, \boldsymbol{R}^{*}\right)$ is an equilibrium iff $\eta_{m} \leq k<\eta_{M}$ (Proposition 6 and ensuing discussion).

\section{RESULTS AND DISCUSSION}

In this section, the behavior of the system in the Nash equilibrium for the competition game, in those cases when it is not $(\mathbf{0}, \mathbf{0})$, is explored. Firstly, the dependency of the upper 
bound of the number of active SPs $\left(\eta_{M}\right)$ with the sensitivity and number of users is studied. Secondly, we explore the strategy $\left(R_{i}^{*}, p_{i}^{*}\right)$ and the resulting surpluses in non-trivial symmetric equilibrium and their dependency with the rest of the system parameters. Thirdly, the strategies and surpluses in equilibrium are compared with those that result in the optimal welfare. And fourthly, SPs profits achieved in equilibrium are compared with those obtained when SPs are allowed to cooperate. For all the subsequent study we have set the following parameters: $f_{m}=1 \mathrm{cu}, r_{\max }=1 \mathrm{nbps}, R_{0}=1$ nbps and $p_{0}=1 \mathrm{cu}$.

\section{A. Upper bound of the number of active SPS}

Let us examine first how many service providers enter the market. In Fig. 8, the value of the upper bound of the number of active SPs, $\eta_{M}$, is represented as a function of the user sensitivity, $\alpha$. In Fig. 9, $\eta_{M}$ is represented as a function of the price paid to the NAPs normalized by $M$. Both figures use a logarithmic scale.

The following effects can be observed:

- $\eta_{M}$ decreases significantly as the sensitivity $\alpha$ increases, which means that the greater sensitivity of users, the less room for competing providers. A higher value for $\alpha$ translates into a fiercer competition between service providers. This situation induces a less stable strategic interaction between the providers, which would explain why only equilibria with a lower number of providers exist.

- For $\alpha<2, \eta_{M}$ is greater than 1, implying that there exists an equilibrium with more than one active SP. Additionally, following from (34), which gives the fraction of users who subscribe to each SP, the total fraction of users that subscribe, i.e. the penetration rate, will be $K$ times the penetration rate in the case of the monopoly. In [12], we showed that in a monopolistic scenario the fraction of subscribers is typically low-as a matter of fact, it would tend to zero when $\alpha$ approaches 1 . Our results here show that this limitation can be completely overcome by allowing competition. In Fig. 10, the penetration rate is represented, as a function of $\alpha$, in both a competition and a monopoly setting (the number of users is $M=1000$ ). We observe that the penetration rate in the competitive setting is always higher than that of the monopolistic setting, and is much higher when $\alpha$ approaches 1 .

- $\eta_{M}$ decreases only slightly as $f_{m} / M$ increases. As we will see below, the influence of $f_{m} / M$ and therefore of $M$ on the equilibrium is negligible.

\section{B. Strategy in symmetric equilibrium}

The following figures show in a semilogarithmic scale the variation of the price, the rate, the rate-price ratio, the SPs profits, the users' surplus and the WSNs' surplus at the equilibrium, as a function of the users sensitivity $\alpha$, for different values of the number of users, $M$, (Figs. 11, 13, 15, 17, 19 and 21) and of the number of WSNs, $N$, (Figs. 12, 14, $16,18,20$ and 22). In those plots containing different values

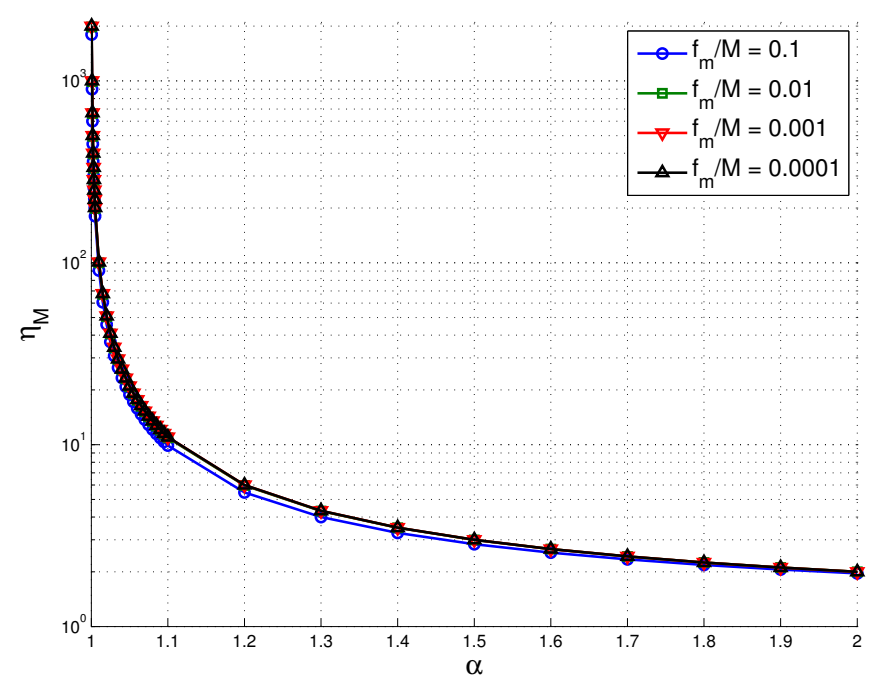

Fig. 8. $\eta_{M}$ for different $f_{m} / M$ values.

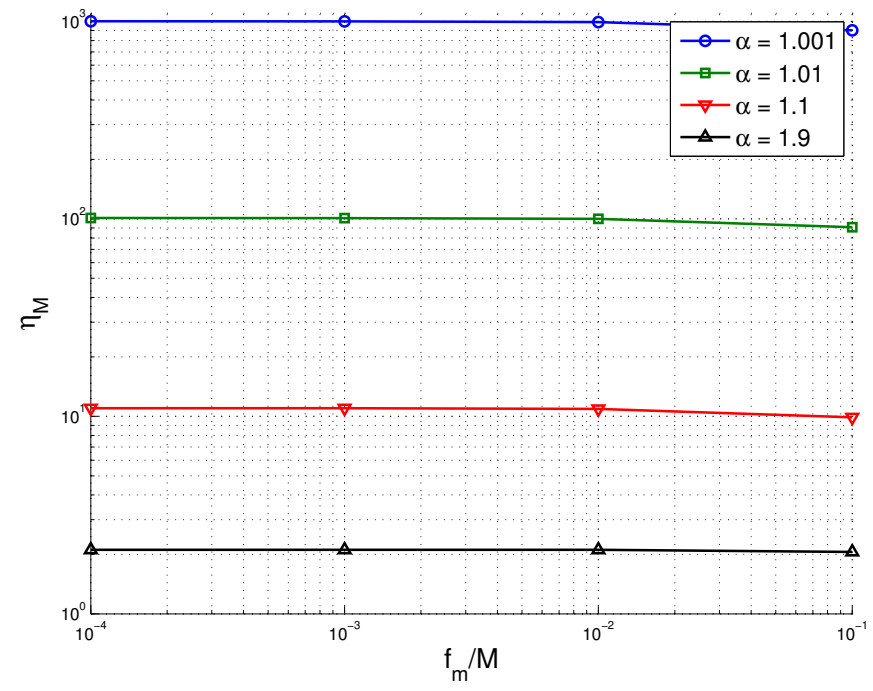

Fig. 9. $\eta_{M}$ for different $\alpha$ values.

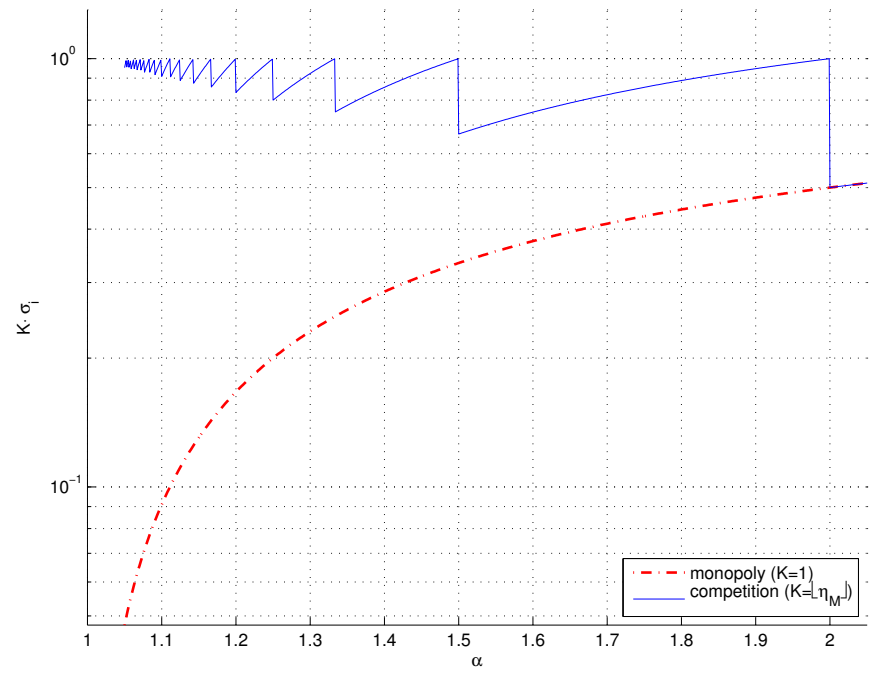

Fig. 10. Penetration rate in competition vs. monopoly. 
of the number of users, $M$, the number of WSNs is set at $N=10$; in those containing different values of the number of WSNs, $N$, the number of users is set at $M=1000$. And the number of SPs is set to $K=9$.

The values represented are computed by numerical evaluation of the expressions derived in Section III. For all the values represented the competition results in a unique symmetric equilibrium.

We can observe the following:

- As regards the equilibrium strategy $\left(R_{i}^{*}, p_{i}^{*}\right)$,

- The price $p_{i}^{*}$ (in cu's) decreases as the sensitivity $\alpha$ increases, since an increased users sensitivity to price induces competition in providers, which results in a fall in the price (Figs. 11 and 12). The influence of $M$ on $p_{i}^{*}$ in negligible, except for very low and likely unrealistic values of $M$ (Fig. 11). On the other hand, it increases when $N$ increases (Fig. 12), since the total rate $R_{m}$ available for providing service to the users increases, which increases the objective part of the users' utility, and this allows the providers to raise the price.

- The rate $R_{i}^{*}$ (in nbps) decreases only slightly with the sensitivity $\alpha$ (Figs. 13 and 14). It approaches the maximum value (which is, being $N=10$ and $r_{\max }=$ $1, R_{m}=5$ ) when $M$ increases (Fig. 13), since the higher $M$ is, the higher the price the providers can pay for the resource, and the higher the rate procured. This happens regardless of the value of $M$, provided that $\mathrm{M}$ is not too low. The plot shows that this is true for values of $\mathrm{M}$ above 100 , but note that a lower number of users would be unrealistic. As regards the dependence on $N$, the rate $R_{i}^{*}$ increases when $N$ increases (Fig. 14), since the total available rate $R_{m}$ also increases.

- Note that the statement that the influence of $M$ on $\left(R_{i}^{*}, p_{i}^{*}\right)$ is negligible holds as long as $M$ is above a critical amount; In the case discussed here the value $M=100$ is above this critical amount. $M$ values lower than this critical value are too low to be realistic, so the influence of $M$ on $\left(R_{i}^{*}, p_{i}^{*}\right)$ is virtually negligible.

- The ratio $R_{i}^{*} / p_{i}^{*}$ (nbps/cu) increases as as the sensitivity $\alpha$ increases (Figs. 15 and 16), since the effect of $\alpha$ is stronger on the price than on the rate. However, there is no effect of $M$ and of $N$ on $R_{i}^{*} / p_{i}^{*}$, which implies that the fraction of users that subscribe to each provider $\sigma_{i}$ remains unchanged (see (13)) and also the total fraction of subscribers (as anticipated in (34) in Proposition 4.).

- As regards the surplus of each agent, bearing in mind that the equilibrium is symmetric:

- The aggregate profit of all SP's, $\Pi_{S P s}^{(s)}$ (in cu's), given by (17), decreases as the sensitivity $\alpha$ increases (Figs. 17 and 18), since the competition between the providers is fiercer, which translates to reduced profits. As $M$ increases, $\Pi_{S P s}$ increases (Fig. 17). Two effects intervene here: on the one hand, the

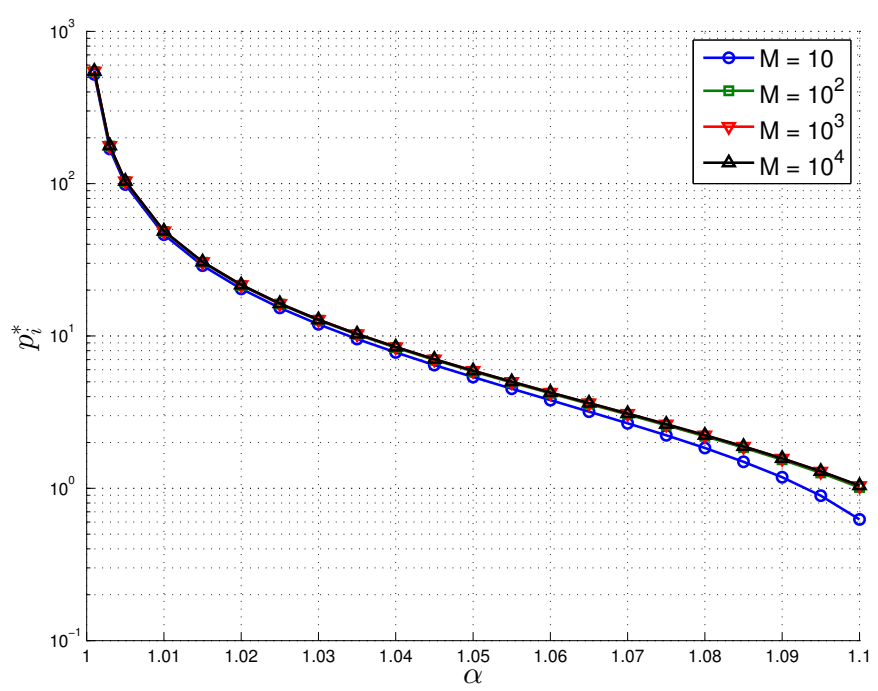

Fig. 11. $p_{i}$ in equilibrium for different $M$ values.

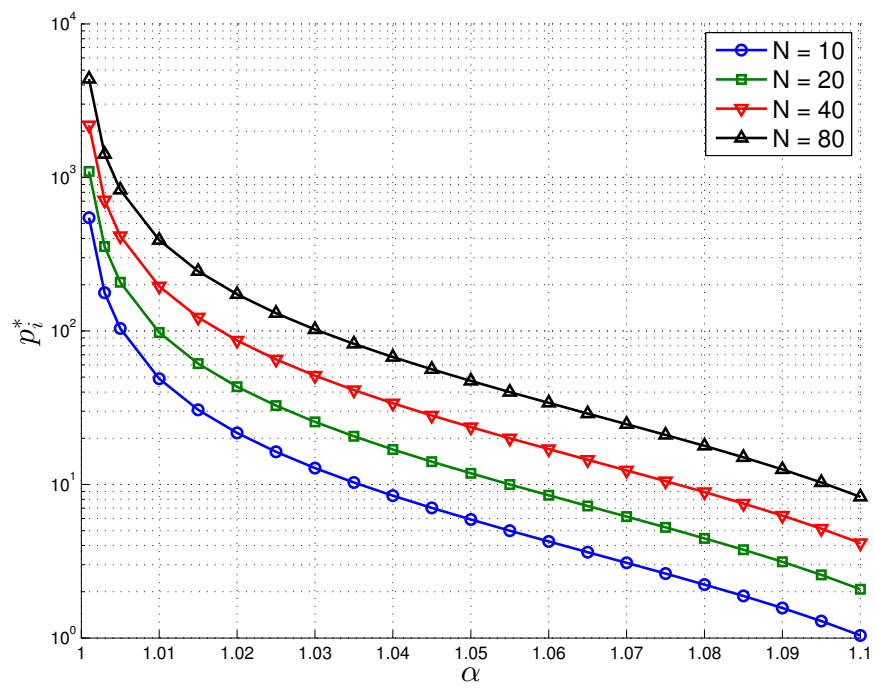

Fig. 12. $p_{i}$ in equilibrium for different $N$ values.

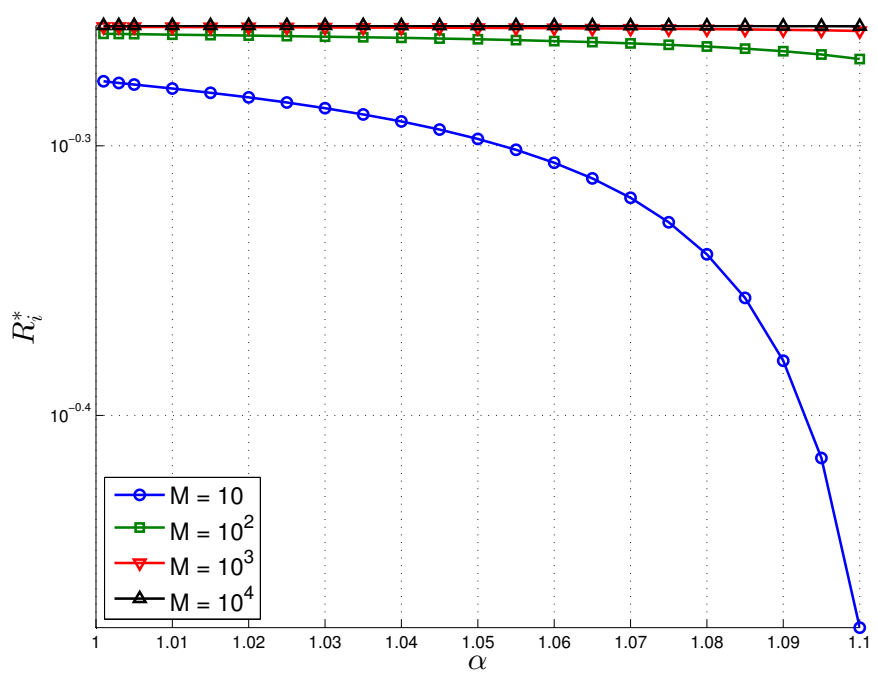

Fig. 13. $R_{i}$ in equilibrium for different $M$ values. 


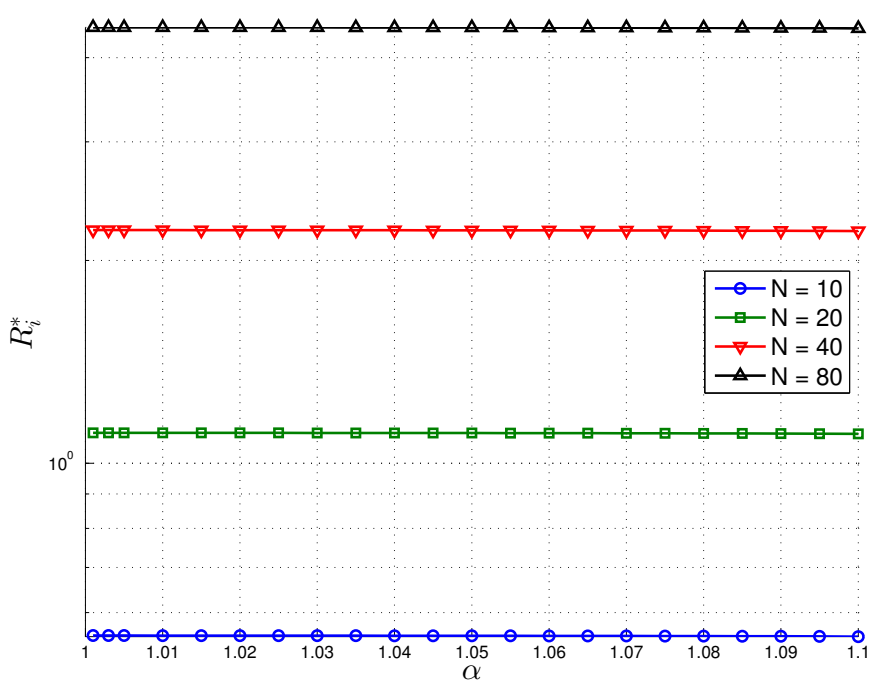

Fig. 14. $R_{i}$ in equilibrium for different $N$ values.

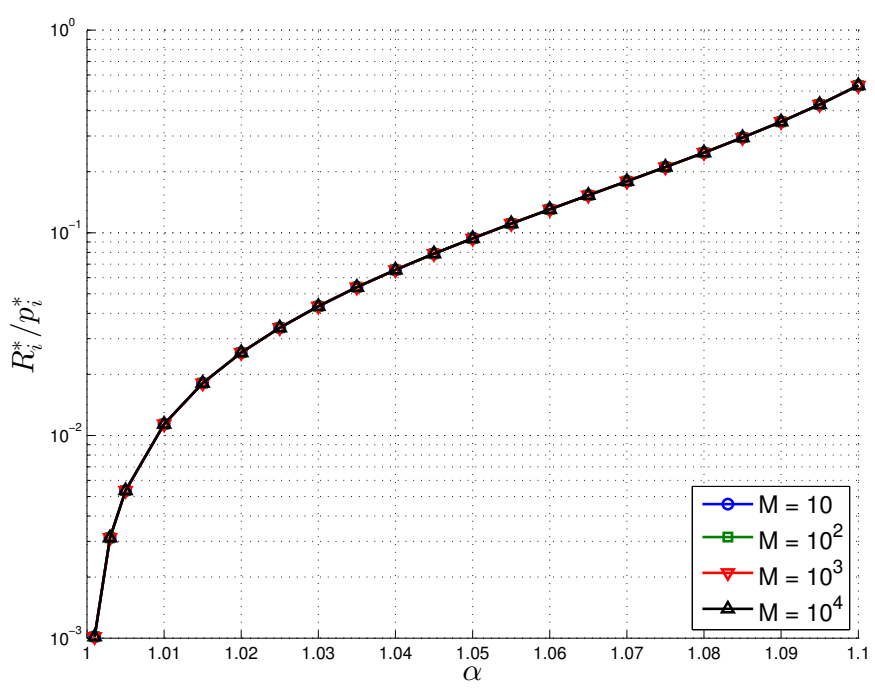

Fig. 15. $R_{i} / p_{i}$ in equilibrium for different $M$ values.

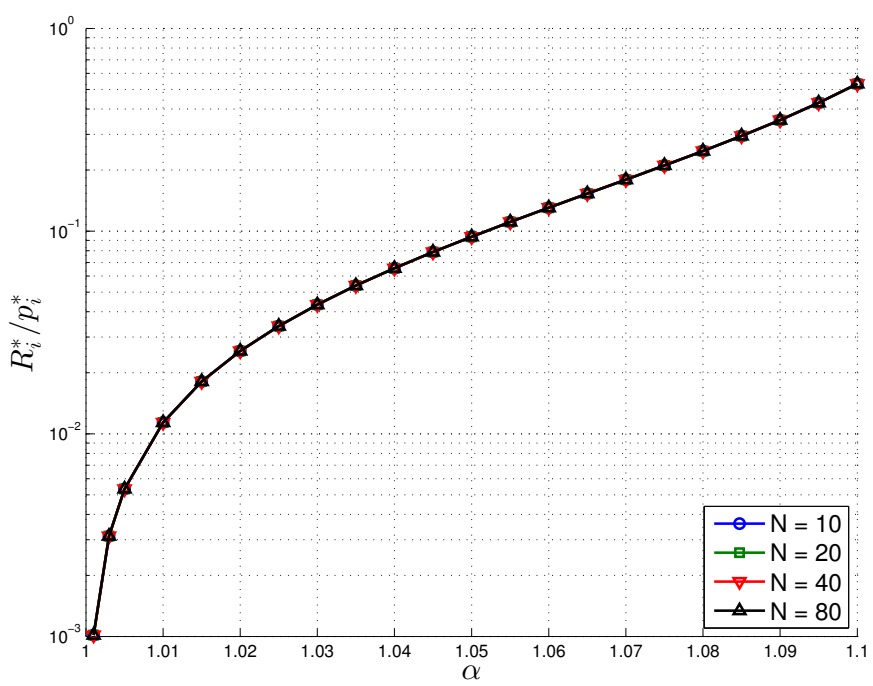

Fig. 16. $R_{i} / p_{i}$ in equilibrium for different $N$ values.

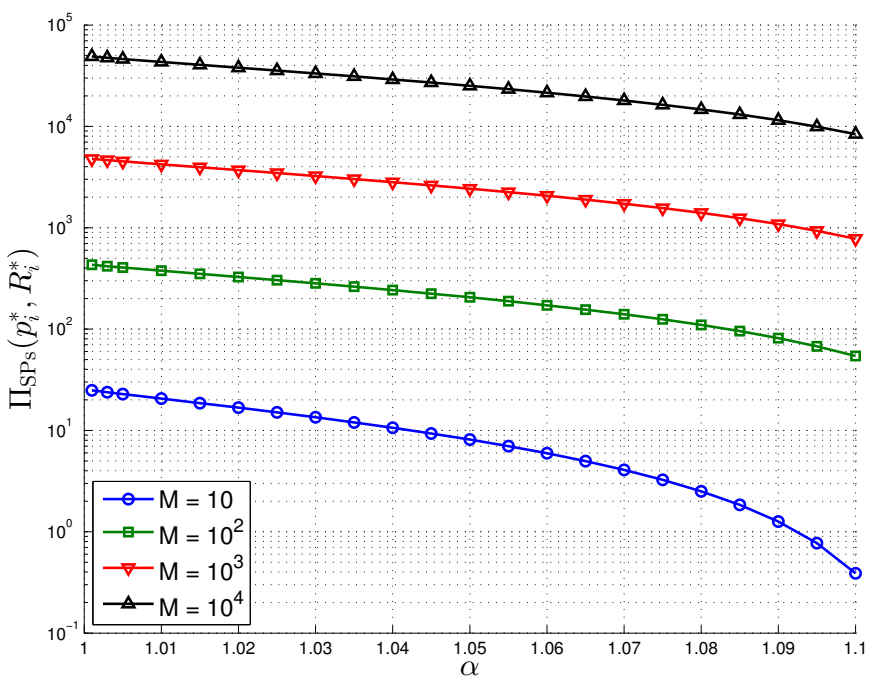

Fig. 17. $\Pi_{S P s}$ in equilibrium for different $M$ values.

revenues $M \sigma_{i} p_{i}$ of each provider increase, since $\sigma_{i}$ remains unchanged-as reasoned above, and the price only varies slightly; on the other hand, the costs $a R_{i}^{*}$ increase just slightly due to slight increments in the information rates $R_{i}$ and consequently in the price $a$. And the first effect dominates. As $N$ increases, $\Pi_{S P s}$ increases (Fig. 18), since the SPs may request more resources at roughly the same price $a$ and therefore may ask users for a higher price.

- Users' surplus $\Pi_{\text {users }}$ (in cu's), given by (15), increases as the sensitivity $\alpha$ increases (Figs. 19 and 20), bearing in mind that, as discussed above, the ratio $R_{i}^{*} / p_{i}^{*}$ increases; indeed, more sensitive users will be able to extract more surplus from the providers. From (33), the number of users, $M$, or the number of WSNs, $N$, have no impact on $R_{i}^{*} / p_{i}^{*}$. Consequently, as it may have been anticipated from (15), we observe a linear growth of $\Pi_{\text {users }}$ with $M$ (Fig. 19) and no variation with $N$ (Fig. 20).

- WSNs' surplus $\Pi_{W S N s}$ (in cu's), given by (11), decreases as the sensitivity $\alpha$ increases (Figs. 21 and 22), since a fiercer competition at the users side translates to a lower price $a$ paid at the WSNs side. $\Pi_{W S N s}$ increases as $M$ increases (Fig. 21), since the rate requested by the providers approaches $R_{m}$ and therefore the price paid $a$ increases significantly (Eq. 10). Finally, $\Pi_{W S N s}$ increases as $N$ increases (Fig. 22), since the total available rate $R_{m}$ increases and also the total rate requested by the providers.

To sum up, assuming that a critical mass of users is present, an equilibrium is reached characterized by the following. First, almost all available rate from the $N$ WSNs is procured to the service providers, which compete in prices for the users. Second, the number of users, $M$, and WSNs, $N$, do not influence the ratio $R_{i}^{*} / p_{i}^{*}$, and therefore more users lead to higher surplus for SPs, WSNs and users, while more WSNs lead to higher surplus just for SPs and WSNs. And third, 


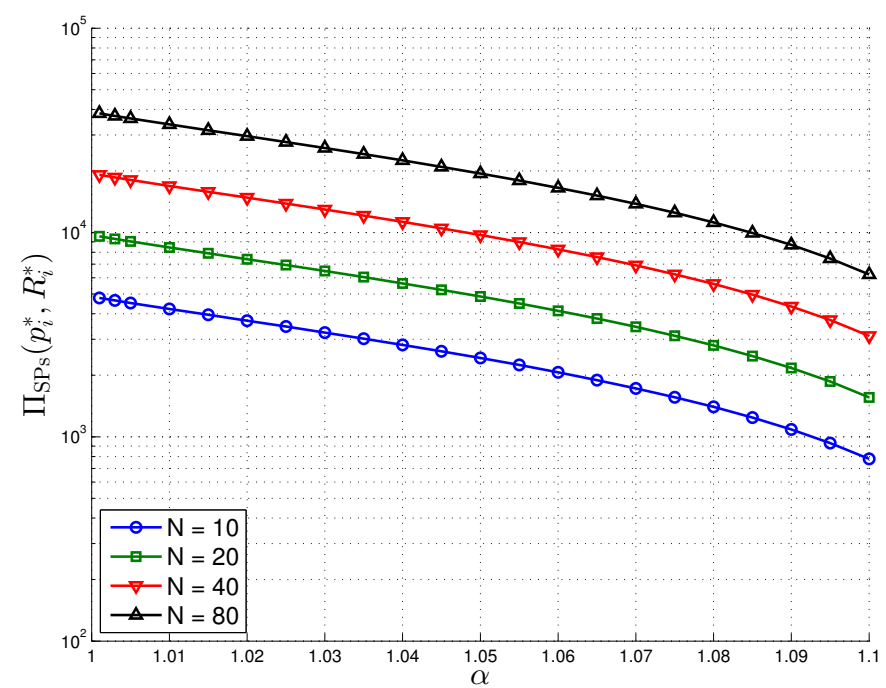

Fig. 18. $\Pi_{S P s}$ in equilibrium for different $N$ values.

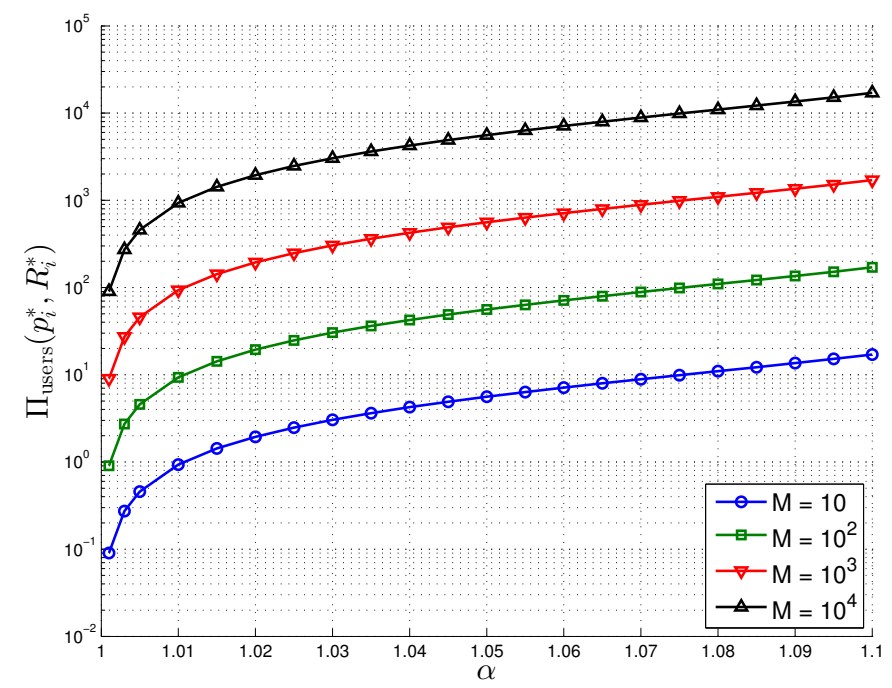

Fig. 19. Users' surplus in equilibrium for different $M$ values.

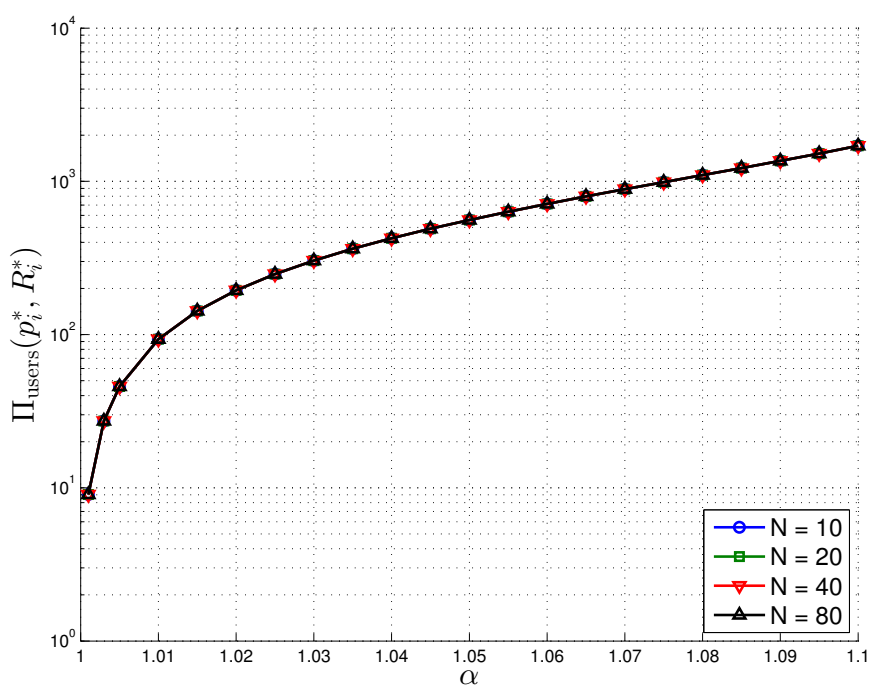

Fig. 20. Users' surplus in equilibrium for different $N$ values.

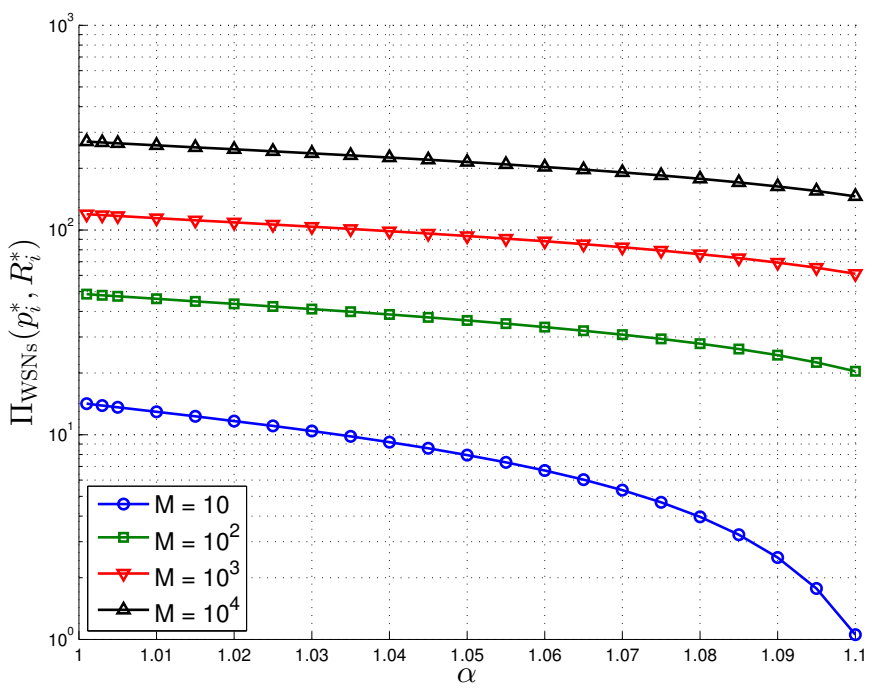

Fig. 21. WSNs' surplus in equilibrium for different $M$ values.

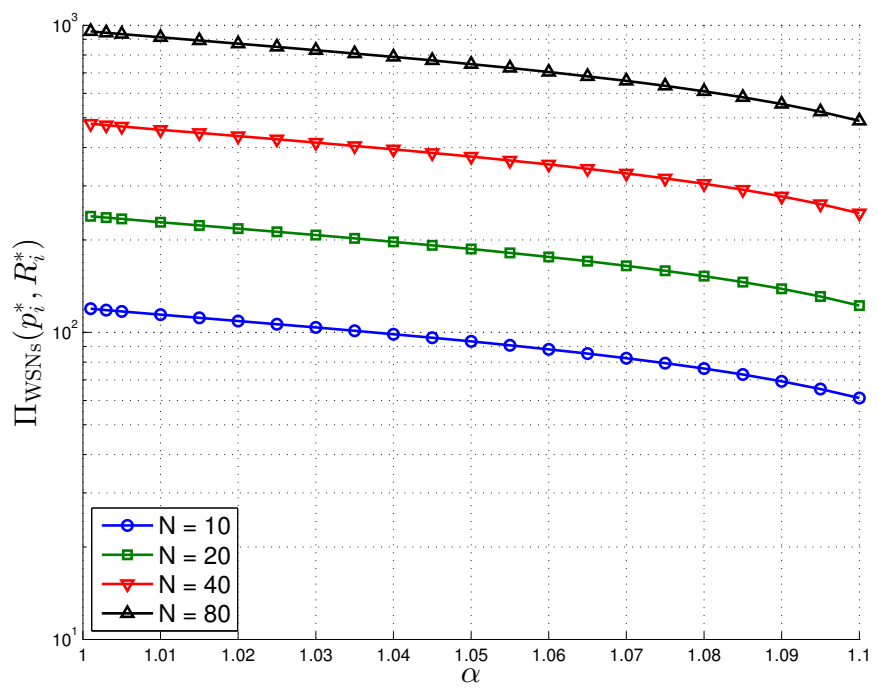

Fig. 22. WSNs' surplus in equilibrium for different $N$ values.

the sensitivity $\alpha$ influences not only the resulting equilibrium, with higher $\alpha$ translating to higher surplus for the users but lower profits for the SPs and for the WSNs, but also the very existence of the equilibrium, since higher $\alpha$ makes equilibrium for numerous providers not feasible.

\section{Social welfare}

The values are now assessed from the point of view of the social welfare.

Social Welfare SW is defined as the sum of the users' surplus, the WSNs' surplus and the SPs' profit. The maximum Social Welfare is called social optimum SO, which can be therefore computed by solving the following maximization problem:

$$
\mathrm{SO}=p_{p_{i}, R_{i} \geq 0,} \max _{\sum_{i=1}^{K} R_{i}<R_{m}} \operatorname{SW}\left(R_{1}, \ldots, R_{K}, p_{1}, \ldots, p_{K}\right) .
$$


Assume that $\left(p_{i}, R_{i}\right) \neq(0,0)$ for $k$ providers. By means of numerical optimization, we have found that the social optimum is achieved at a symmetric $2 K$-tuple with identical $R_{i}$ 's and identical $p_{i}$ 's. Therefore, in order to compute the social optimum, we only need the SW expression for the symmetric case. The expressions for the users' surplus and the SPs' profit in the symmetric case and for the WSNs' surplus have been obtained in (15), (17) and (11), respectively. The Social Welfare in the symmetric case, $\mathrm{SW}^{(s)}$, is then

$$
\begin{aligned}
& \mathrm{SW}^{(s)}\left(R_{i}, p_{i}\right)= \\
& \quad \Pi_{\mathrm{users}}^{(s)}\left(R_{i}, p_{i}\right)+\Pi_{W S N s}\left(R_{i}, p_{i}\right)+\Pi_{S P s}^{(s)}\left(R_{i}, p_{i}\right),
\end{aligned}
$$

and the social optimum is

$$
\mathrm{SO}=\max _{\left(p_{i}, R_{i}\right) \in\{(0,0)\} \cup\left\{(0, \infty) \times\left(0, R_{m} / K\right)\right\}} \mathrm{SW}^{(s)}\left(p_{i}, R_{i}\right) .
$$

In order to measure the loss of efficiency due to the selfish behavior of service providers, we will use the price of anarchy PoA, defined as [27]:

$$
\mathrm{PoA} \equiv \frac{\mathrm{SO}}{\mathrm{SW}^{(s)}\left(p_{i}^{*}, R_{i}^{*}\right)},
$$

where a symmetric $2 K$-tuple with identical $R_{i}^{*}$ 's and identical $p_{i}^{*}$ 's is a (Nash) equilibrium point. The social optimum has been computed by numerical maximization while the social welfare at the equilibrium has been computed using the analytical expressions.

In all of the following plots, the users sensitivity is set at $\alpha=1.1$. For the parameter values used, $\eta_{M}=10.993$ and $\eta_{m}=10.986$, so that the competition results in a unique symmetric equilibrium for a number of SPs $K \leq 10$. And the following values are used: $M=1000$ and $N=10$. Fig. 23 (respectively, Fig. 24) shows the equilibrium and optimum $R_{i}$ (respectively, $p_{i}$ ) as a function of the number of providers $K$. Figs. 25, 26, 27 and 28 show the equilibrium and social optimum $\Pi_{\text {users }}, \Pi_{S P s}, \Pi_{W S N s}$, and $S W$, respectively, as a function of $K$. All graphs are plot in a semilogarithmic scale, except Fig. 26, which is plot in a linear scale.

Fig. 23 shows that, both in equilibrium and in the optimal welfare, $R_{i}$ is close to the maximum $\left(R_{m} / K\right)$, that is, almost all the available information rate is supplied to the providers, with a slight difference in favor of the social optimum. This is best seen in Fig. 29, in which $R_{i}$ is normalized relative to the $K$-th fraction of the available rate $\left(R_{m} / K\right)$. This figure shows that $R_{i} K / R_{m}$ in equilibrium presents a maximum at approximately half the range of $K$. We have experimentally found that such behavior, hard to explain because of the complexity of the system equilibria, remains qualitatively the same for a wide range of values of parameters $\alpha, M$ and $N$.

In Fig. 24 it can be seen that price $p_{i}$ charged to users decreases rapidly with the number of competing providers $K$, while it decreases much more slowly for the optimal welfare.

The consequences of this behavior can be seen in Figs. 25, 26, 27 and 28. On the one hand, in the competition case, as $K$ increases, the profits shifts from the providers (Fig. 26) to the users (Fig. 25), while the WSNs' surplus only increases slightly up to a maximum when $K=5$, and then decreases but not as dramatically as the SPs profits (Fig. 27). On the other

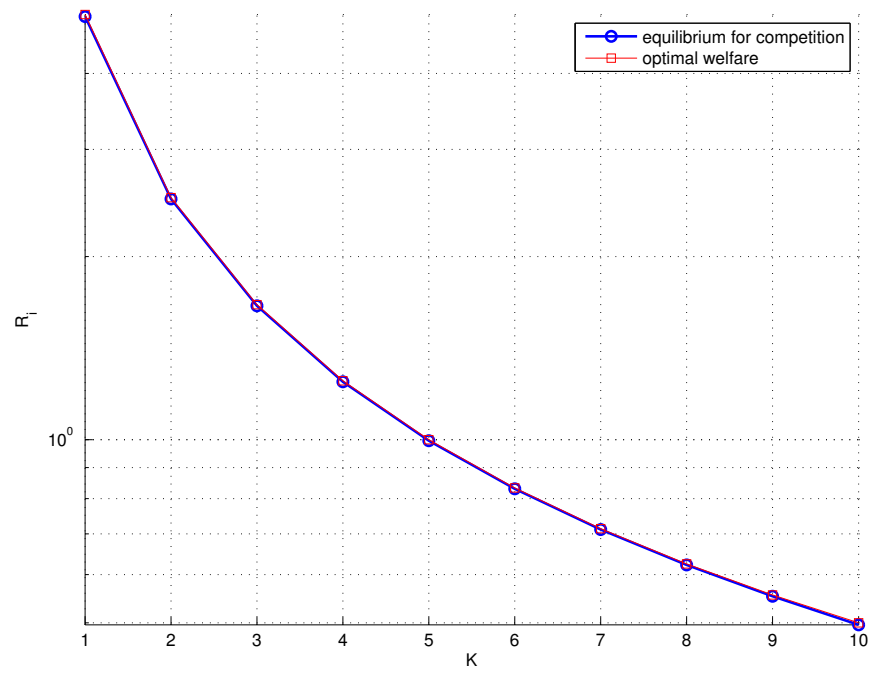

Fig. 23. $R_{i}$ in equilibrium and in optimal welfare.

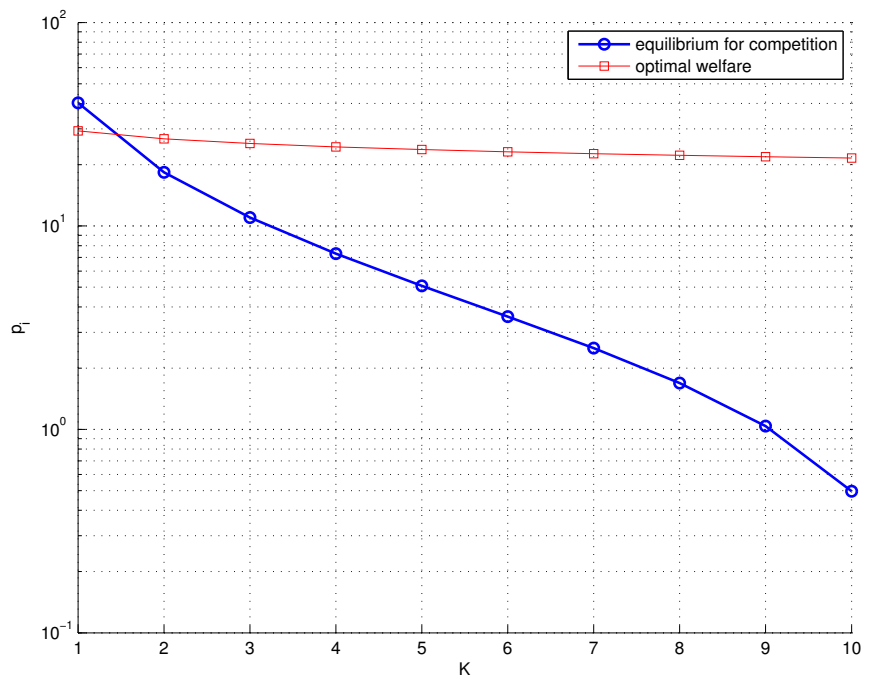

Fig. 24. $p_{i}$ in equilibrium and in optimal welfare.

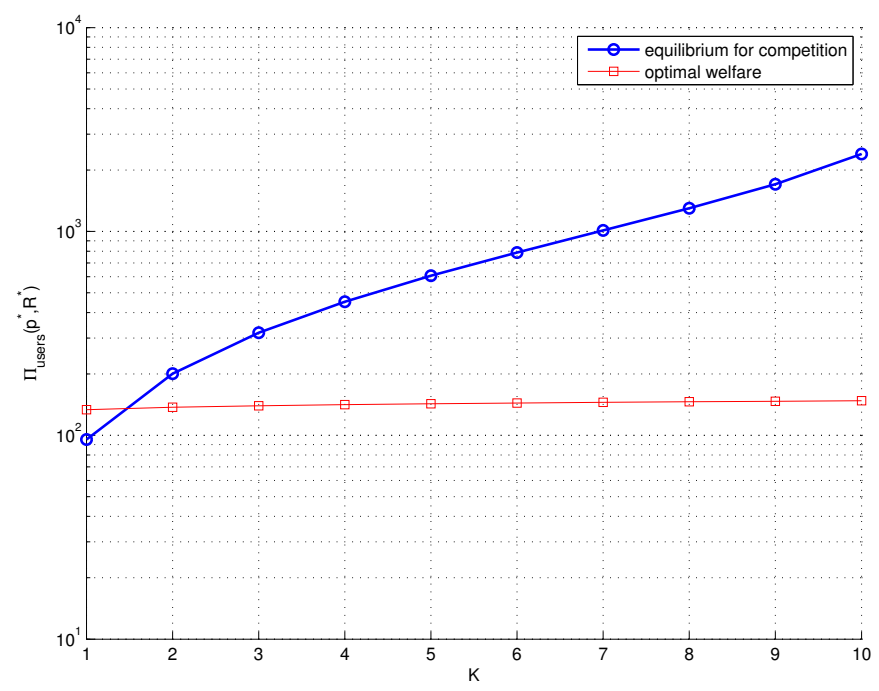

Fig. 25. Users' surplus in equilibrium and in optimal welfare. 


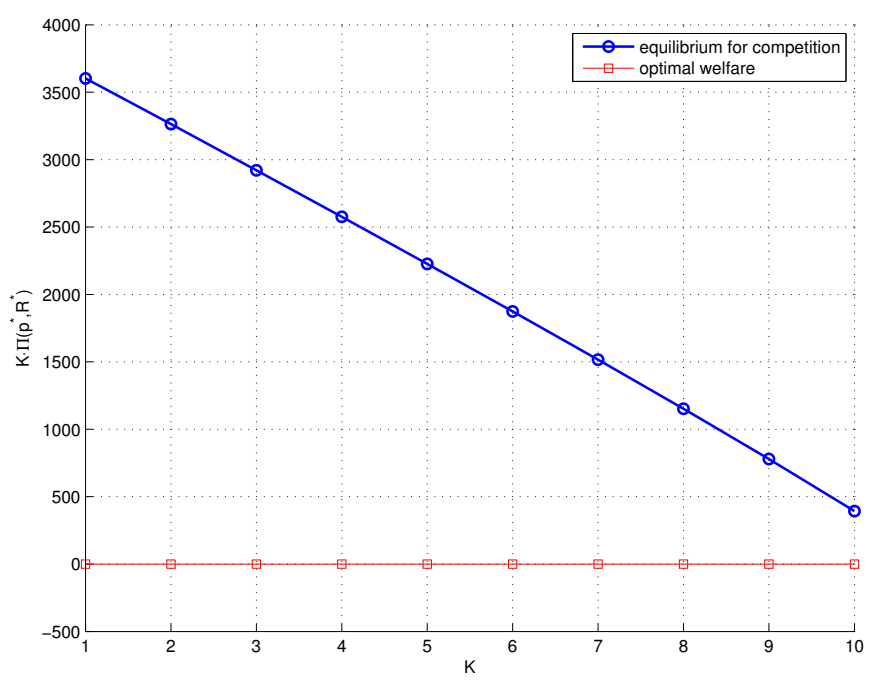

Fig. 26. SPs profits in equilibrium and in optimal welfare.

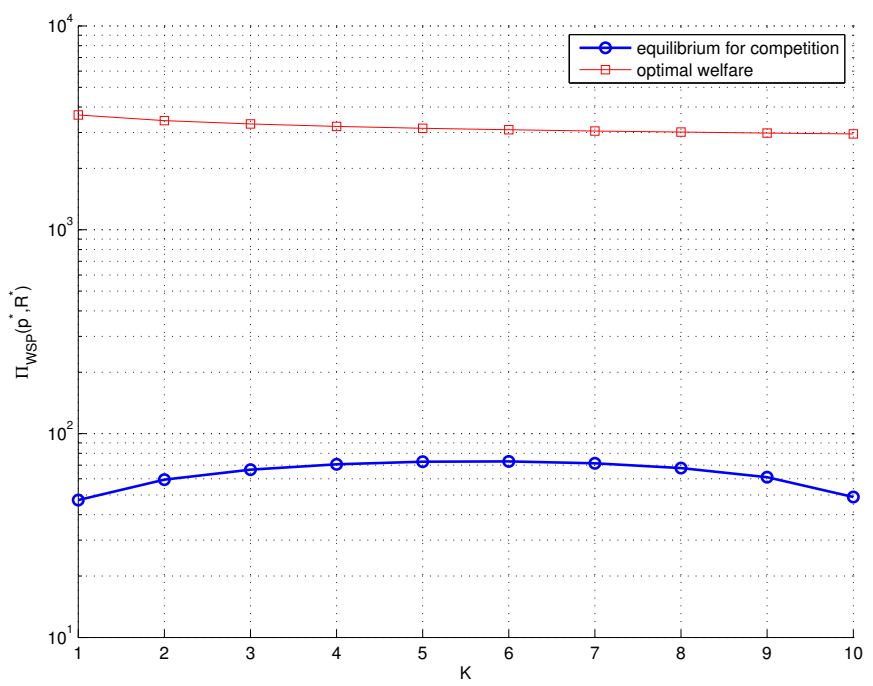

Fig. 27. WSNs' surplus in equilibrium and in optimal welfare.

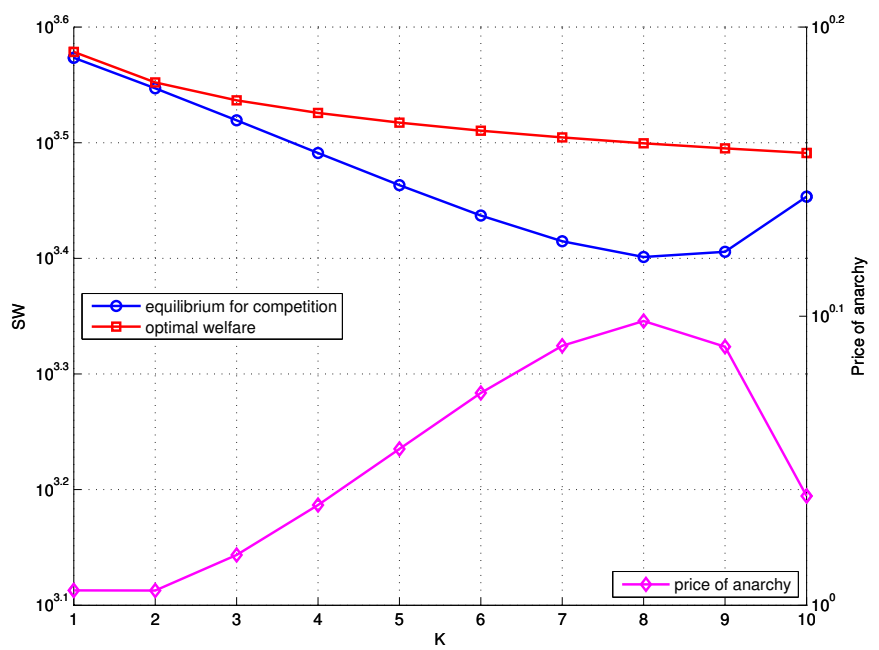

Fig. 28. Social welfare in equilibrium and in optimal welfare, and price of anarchy.

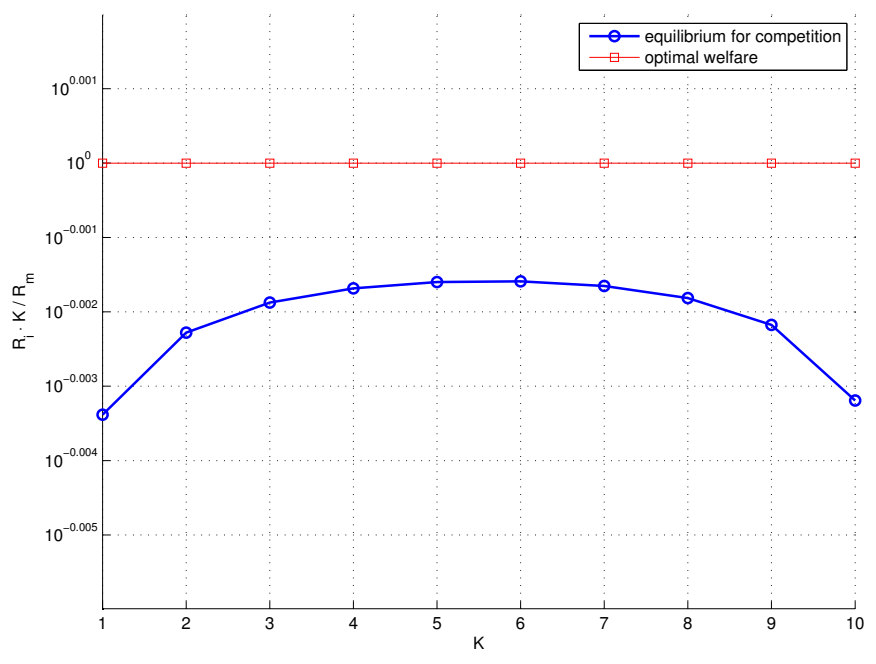

Fig. 29. $R_{i} \cdot K / R_{m}$ in equilibrium and in optimal welfare.

hand, in the social optimum case, SPs profits are zero (Fig. 26), which means that maximum social welfare implies setting intermediation profits to zero. As $K$ increases, users' surplus increases slightly (Fig. 25) and WSNs' surplus decreases (Fig. 27), resulting in a decrease in the social welfare (Fig. 28), up to the value $K=8$. As expected by the definition of social optimum, the social welfare is greater for the social optimum than for the competition (Fig. 28), and the gap between them is roughly greater when $K$ increases, since the efficiency loss due to the non-cooperative strategic interaction increases when the number of players increases. This is also seen in the curve of the price of anarchy (Fig. 28), defined as the the ratio between the optimal social welfare and the social welfare in equilibrium. Again, this applies up to the value $K=8$, and from this value the gap is reduced; this particularity deserves a deeper discussion that is deferred for further study.

\section{CONCLUSiOnS}

The following conclusions can be drawn from our study:

- An intermediation business model is proposed for WSNbased service provision, which is sustainable provided that the user sensitivity to the sensing-rate-to-price ratio is not negligible. And in this situation the number of active service providers is upper bounded by a value that depends on the sensitivity and on the market size.

- The operation of such a market is shown to be able to use all available information generated by the WSNs, and therefore to provide a near-maximum quality service.

- The sensitivity of users toward the information-rate-toprice ratio has been shown to be of utmost importance, not only because influences the distribution of the surplus between the producers, the intermediaries and the users, but also because it determines the existence of the equilibrium in the competition game.

- Compared to the social optimum, the competition outcome has been shown to match the utilization of the resources, to deliver a better surplus to the users and to the intermediaries, but to leave the WSNs worse off. 


\section{REFERENCES}

[1] D. Evans, "The internet of things. how the next evolution of the internet is changing everything," White paper, vol. 2011, no. 4, pp. 1-11, 2017.

[2] J.-M. Bohli, C. Sorge, and D. Westhoff, "Initial observations on economics, pricing, and penetration of the internet of things market," $A C M$ SIGCOMM Computer Communication Review, vol. 39, no. 2, pp. 50-55, 2009.

[3] C. Perera, A. Zaslavsky, P. Christen, and D. Georgakopoulos, "Sensing as a service model for smart cities supported by internet of things," Transactions on Emerging Telecommunications Technologies, vol. 25, no. 1, pp. 81-93, 2014.

[4] C. Wood, "Smart cities: Understanding the untapped value of sensor data," Government Technology, 2014. [Online]. Available: http://www.govtech.com/data/ Smart-Cities-Understanding-the-Untapped-Value-of-Sensor-Data.html

[5] M. M. Islam, M. M. Hassan, G.-W. Lee, and E.-N. Huh, "A survey on virtualization of wireless sensor networks," Sensors, vol. 12, no. 2, pp. 2175-2207, 2012.

[6] I. Khan, F. Belqasmi, R. Glitho, N. Crespi, M. Morrow, and P. Polakos, "Wireless sensor network virtualization: early architecture and research perspectives," Network, IEEE, vol. 29, no. 3, pp. 104-112, 2015.

[7] X. Vives, Oligopoly pricing: old ideas and new tools. MIT press, 2001.

[8] E. Fleisch, M. Weinberger, and F. Wortmann, "Business models and the internet of things," in Interoperability and Open-Source Solutions for the Internet of Things. Springer, 2015, pp. 6-10.

[9] T. Keskin and D. Kennedy, "Strategies in smart service systems enabled multi-sided markets: Business models for the internet of things," in System Sciences (HICSS), 2015 48th Hawaii International Conference on. IEEE, 2015, pp. 1443-1452.

[10] L. Guijarro, V. Pla, J. Vidal, and M. Naldi, "Maximum-profit two-sided pricing in service platforms based on wireless sensor networks," Wireless Communications Letters, IEEE, vol. 5, no. 1, pp. 8-11, 2016.

[11] L. Guijarro, M. Naldi, V. Pla, and J. R. Vidal, "Pricing of wireless sensor data on a centralized bundling platform," in 23nd International Conference on Telecommunications (ICT). Thessaloniki: IEEE, 2016.

[12] _ _Economic analysis of a centralized brokering platform for wireless sensor data," in 14th Annual IEEE Consumer Communications and Networking Conference (CCNC), Las Vegas, 2017, accepted.

[13] D. Niyato, D. T. Hoang, N. C. Luong, P. Wang, D. I. Kim, and Z. Han, "Smart data pricing models for the internet of things: a bundling strategy approach," IEEE Network, vol. 30, no. 2, pp. 18-25, 2016.

[14] D. Niyato, X. Lu, P. Wang, D. I. Kim et al., "Economics of internet of things: An information market approach," IEEE Wireless Communications, p. 137, 2016.

[15] D. Ardagna, B. Panicucci, and M. Passacantando, "Generalized Nash equilibria for the service provisioning problem in cloud systems," Services Computing, IEEE Transactions on, vol. 6, no. 4, pp. 429-442, 2013.

[16] M. Palattella, M. Dohler, A. Grieco, G. Rizzo, J. Torsner, T. Engel, and L. Ladid, "Internet of things in the 5G era: Enablers, architecture and business models," Selected Areas in Communications, IEEE Journal on, vol. 34, no. 3, pp. 510-527, 2016.

[17] V. Sachidananda, A. Khelil, and N. Suri, "Quality of information in wireless sensor networks: A survey," in Information Quality (ICIQ), 2010 15th International Conference on. ICIQ, 2010, pp. 193-207.

[18] N. Russo, R. Morgus, S. Morris, and D. Kehl, "The cost of connectivity," New American Foundation, Open Technology Institute, 2014.

[19] A. Odlyzko, "Will smart pricing finally take off?" in Smart data pricing, S. Sen, C. Joe-Wong, S. Ha, and M. Chiang, Eds. John Wiley \& Sons, 2014, pp. 3-33.

[20] A. R. Syversveen, "Noninformative bayesian priors. interpretation and problems with construction and applications," Preprint Statistics, vol. 3, 1998.

[21] L. Guijarro, M. Naldi, V. Pla, and J. R. Vidal, "Optimal pricing strategy for a wireless sensor data broker under a zipf-distributed sensing rate offer," in 27th Annual IEEE International Symposium on Personal, Indoor, and Mobile Radio Communications (PIMRC), Valencia, 2016.

[22] M. E. Ben-Akiva and S. R. Lerman, Discrete choice analysis: theory and application to travel demand. MIT press, 1985, vol. 9.

[23] P. Maillé and B. Tuffin, Telecommunication network economics: from theory to applications. Cambridge University Press, 2014.

[24] P. Reichl, B. Tuffin, and R. Schatz, "Logarithmic laws in service quality perception: where microeconomics meets psychophysics and quality of experience," Telecommunication Systems, 2011, pp. 1-14.

[25] K. E. Train, Discrete choice methods with simulation. Cambridge university press, 2009.
[26] L. Guijarro, V. Pla, J. R. Vidal, and M. Naldi, "Game theoretical analysis of service provision for the internet of things based on sensor virtualization," Universitat Politècnica de València, Tech. Rep. TELPOL101, 2016. [Online]. Available: http://personales.upv.es/lguijar/ pubs/TELPOL101-TR.pdf

[27] E. Koutsoupias and C. Papadimitriou, "Worst-case equilibria," in Annual Symposium on Theoretical Aspects of Computer Science. Springer, 1999, pp. 404-413.

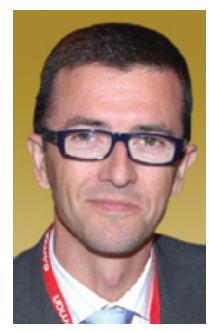

Luis Guijarro received the Ph.D. degree in Telecommunication engineering from the Universitat Politècnica de València (UPV), Spain in 1998. He is an Associate Professor in Telecommunications Policy at the UPV. He researched in traffic management in ATM networks and in e-Government, and collaborated with the Jean Monnet Chair on Telecommunications and Information Society Policy at the European Union, based in the UPV, and published the book The Electronic Communications Policy of the European Union. His current research is focused on economic modeling of telecommunication service provision. $\mathrm{He}$ has contributed in the areas of peer-to-peer interconnection, cognitive radio networks, search engine neutrality, and wireless sensor networks.

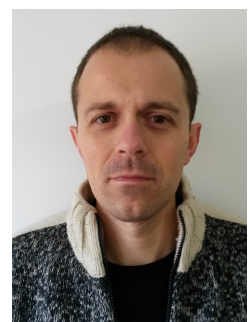

Vicent Pla received the M.E. and Ph.D. degrees in Telecommunications from the Universitat Politècnica de València (UPV), Spain, and the B.Sc in Mathematics from the Universidad Nacional de Educación a Distancia (UNED), Spain. In December 1999, he joined the Department of Communications at the UPV, where he is currently an Associate Professor. His research interests lie primarily in the area of modeling and performance analysis of communication networks. During the past few years, most of his research activity has focused on resource management in wireless networks. In these areas he has published numerous papers in refereed journals and conference proceedings, and has been an active participant in several national and European research projects.

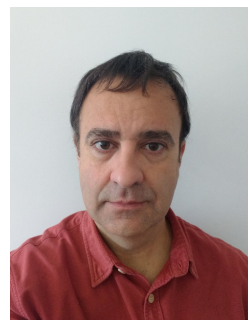

José-Ramón Vidal is currently an Associate Professor in Telematics at the Higher Technical School of Telecommunication Engineering of the Universitat Politècnica de València (UPV), València, Spain. He obtained the PhD in Telecommunication Engineering from the UPV. His current research interest is focused on the area of application of game theory to resource allocation in cognitive radio networks and to economic modeling of telecommunication service provision. In these areas he has published several papers in refereed journals and conference

proceedings

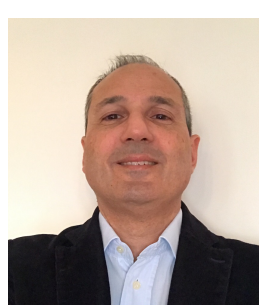

Maurizio Naldi (SM00) graduated cum laude in 1988 in Electronic Engineering at the University of Palermo and then received his Ph.D. in Telecommunications Engineering from the University of Rome "Tor Vergata". After graduation he pursued an industrial career, first at Selenia as a radar designer (1989-1991), and then in the Network Planning Departments of Italcable (1991-1994), Telecom Italia (1995-1998), and WIND (1998-2000) where he was appointed Head, Traffic Forecasting \& Network Cost Evaluation Group. In the 1992-2000 period he was active in the standardization bodies (ETSI and ITU), in particular as Associate Rapporteur for Broadband Traffic Measurements and Models at ITU Study Group 2. Since 2000 he is with the University of Rome at Tor Vergata, where he is now a tenured Aggregate Professor. His research interests lie at the intersection of Computer Science and Economics, mainly in Network Economics. He is a Senior Editor of Electronic Commerce Research and Applications (Elsevier). 